\title{
ISOSPECTRALITY FOR GRAPH LAPLACIANS UNDER THE CHANGE OF COUPLING AT GRAPH VERTICES: NECESSARY AND SUFFICIENT CONDITIONS
}

\author{
YULIA ERSHOVA, IRINA I. KARPENKO, AND ALEXANDER V. KISELEV
}

\begin{abstract}
Laplace operators on finite compact metric graphs are considered under the assumption that matching conditions at graph vertices are of $\delta$ and $\delta^{\prime}$ types. Assuming rational independence of edge lengths, necessary and sufficient conditions of isospectrality of two Laplacians defined on the same graph are derived and scrutinized. It is proved that the spectrum of a graph Laplacian uniquely determines matching conditions for "almost all" graphs.
\end{abstract}

\section{INTRODUCTION}

In the present paper we study the so-called quantum graph, i.e., a metric graph $\Gamma$ with an associated second-order differential operator acting in Hilbert space $L^{2}(\Gamma)$ of square summable functions with an additional assumption that functions belonging to the domain of the operator are coupled by certain matching conditions at graph vertices. These matching conditions reflect the graph connectivity and usually are assumed to guarantee self-adjointness of the operator. Recently these operators have attracted a considerable interest of both physicists and mathematicians due to a number of important physical applications, e.g., to the study of quantum wavequides. Extensive literature on the subject is surveyed in, e.g., [22, 6].

The present paper is devoted to the study of an inverse spectral problem for Laplace operators on compact metric graphs. The inverse

2000 Mathematics Subject Classification. Primary 47A10; Secondary 34A55, 81Q35.

Key words and phrases. Quantum graphs, Laplace operator, inverse spectral problem, boundary triples, isospectral graphs.

The third authors' work was partially supported by the RFBR, grant no. 12-0100215-a. 
problem we have in mind is this: does the spectrum of a graph Laplacian uniquely determine matching conditions at graph vertices (and therefore, the operator itself)?

The related problem of whether the spectrum determines the graph topology (in the case when matching conditions are assumed to be standard, or Kirchhoff) has been studied extensively. To name just a few, we would like to mention the pioneering works [29, 19, 16] and later contributions [23, 25, 2, 17]. These papers utilize an approach to the problem based on the so-called trace formula which relates the spectrum of a quantum graph to the set of closed paths on the underlying metric graph. Different approaches to the same problem were developed, e.g., in [28, 4, 5], see also [24] for the analysis of yet another related problem.

On the other hand, the inverse problem we study in the present paper has to the best of our knowledge surprisingly attracted much less interest. We believe it was first treated in [8]. In the cited paper the square of self-adjoint operator of the first derivative was considered (thus disallowing both $\delta-$ and $\delta^{\prime}-$ coupling at graph vertices) on a subset of metric graphs. Then, after being mentioned in [25, 2], it was treated in [3], but only in the case of star graphs. In our papers [10, 11, 12 we suggested an approach based on the theory of boundary triples, leading to the asymptotic analysis of Weyl-Titchmarsh $M$-function of the graph.

In the present paper we consider the case of a general connected compact finite metric graph (in particular, this graph is allowed to possess both cycles and loops), but only for two possible classes of matching conditions at graph vertices. Namely, each vertex is allowed to have matching of either $\delta$ or $\delta^{\prime}$ type (see Section 2 for definitions). The named two classes singled out by us prove to be physically viable [13, 14]. We further restrict ourselves to the case when all edge lengths of the graph $\Gamma$ are assumed to be rationally independent. This case is known to yield the most complete results in the inverse topology problem as well.

The results we present demonstrate that most graphs prevent nontrivial isospectral configurations. Moreover, isospectrality turns out to be only possible in "simple" graphs (chains or simple cycles). Nevertheless, the answer to the problem of isospectrality is not trivial: in particular, we demonstrate that there exists a stark difference between graph Laplacians with matching conditions of a "pure" type (be it $\delta$ or $\left.\delta^{\prime}\right)$ and those with matching conditions of "mixed" type. 
We point out that techniques developed in the present paper also allow for the treatment of inverse topology problem; moreover, consideration of Schrödinger operators on metric graphs is also possible (see, e.g., [11, 12]). The corresponding results will be published elsewhere.

The approach suggested is based on the celebrated theory of boundary triples [15]. The notion of a generalized Weyl-Titchmarsh $M$ function for a properly chosen maximal operator allows us to reduce the study of spectra of graph Laplacians to the study of "zeroes" of the corresponding finite-dimensional analytic matrix function. The results are then obtained by asymptotic analysis.

The results of the present paper are in a sense complete, i.e., can be used to ascertain the absence of isospectral configurations or otherwise for any compact metric graph; yet at the same time a number of meaningful questions are left open.

The paper is organized as follows.

Section 2 introduces the notation and contains a brief summary of the material on the boundary triples used by us in the sequel as well as an explicit formula (derived in [12]) for the Weyl-Titchmarsh $M$-function written down in what we would like to think of as its "natural" form.

In Section 3 we derive conditions necessary and sufficient for isospectrality of a pair of graph Laplacians under the additional restriction that all edge lengths of the graph are rationally independent.

Finally, in Section 4 we justify the procedure of reducing the problem of isospectrality to the same for a pair of Laplacians defined on a "smaller" graph which is nothing but the original one, "trimmed" in a proper way.

In our view, the main results of the paper are contained in the following assertions:

- Theorem 3.5, which, although unsuitable for most applications, serves not only as a cornerstone of our further analysis, but also allows to give complete solution to the problem of isospectrality for sufficiently simple graphs.

- Theorem 3.8 ascertains that any non-trivial graph with all edges (except loops) of mixed type, i.e., with any two adjacent vertices having different $\left(\delta\right.$ and $\left.\delta^{\prime}\right)$ types of coupling, disallows isospectral configurations of coupling constants provided that the latter are all assumed to be non-zero.

- Theorem 4.8 then provides the ultimate answer to the problem of isospectrality for graphs with all edges of mixed type, stating that if the graph is clean [26] and the coupling constants 
are allowed to zero out, the only non-trivial graph permitting isospectral configurations is the chain $A_{3}$.

- Theorem 4.1tells one that under the assumption that two graph Laplacians are isospectral on a graph $\Gamma$, the corresponding pair of Laplacians has to be isospectral on a smaller ("trimmed", see Definition 3) graph $\Gamma^{(e)}$ with both sets of coupling constants changing in a controllable way. This result shows that for an arbitrary graph one can indeed reduce the study of isospectrality phenomenon to the same on either an arbitrarily simple graph (if all graph vertices have the same type of coupling) or a graph with all edges of mixed type, the answer in both cases being either known or easy to obtain.

- Finally, the balancing conditions (14) of Theorem 3.8 and the assertion of Theorem 4.7 can be viewed as "local" uniqueness results. In particular, Theorem 4.7 ascertains that on a clean graph $\Gamma$ isospectrality of two graph Laplacians immediately yields uniqueness of coupling constants at all vertices, adjacent to the one where coupling constants of both Laplacians are known to zero out simultaneously; the balancing conditions (14) guarantee in a sense the converse, allowing to ascertain the uniqueness of coupling constants at a given vertex based on equality of coupling constants at adjacent vertices.

\section{PRELiminaries}

We call $\Gamma=\Gamma\left(\mathbf{E}_{\boldsymbol{\Gamma}}, \sigma\right)$ a finite compact metric graph, if it is a collection of a finite non-empty set $\mathbf{E}_{\boldsymbol{\Gamma}}$ of compact intervals $e_{j}=\left[x_{2 j-1}, x_{2 j}\right]$, $j=1,2, \ldots, n$, called edges, and of a partition $\sigma$ of the set of endpoints $\left\{x_{k}\right\}_{k=1}^{2 n}$ into $N$ classes, $\mathbf{V}_{\boldsymbol{\Gamma}}=\bigcup_{m=1}^{N} V_{m}$. The equivalence classes $V_{m}$, $m=1,2, \ldots, N$ will be called vertices and the number of elements belonging to the set $V_{m}$ will be called the valence of the vertex $V_{m}$ (denoted $\operatorname{deg} V_{m}$ ).

With a finite compact metric graph $\Gamma$ we associate Hilbert spaces

$$
L_{2}(\Gamma)=\bigoplus_{j=1}^{n} L_{2}\left(\Delta_{j}\right) \text { and } W_{2}^{2}(\Gamma)=\bigoplus_{j=1}^{n} W_{2}^{2}\left(\Delta_{j}\right) .
$$

These spaces obviously do not "feel" the graph connectivity, being the same for each graph with the same number of edges of same lengths.

In what follows, we single out two natural [13] classes of so-called matching conditions which lead to a correctly defined self-adjoint operator on the graph $\Gamma$, namely, matching conditions of $\delta$ and $\delta^{\prime}$ types. In order to describe these, we introduce the following notation. For a 
function $f \in W_{2}^{2}(\Gamma)$, we will use the following definition of the normal derivative $\partial_{n} f\left(x_{j}\right)$ at the endpoints of an interval $e_{k}$ throughout:

$$
\partial_{n} f\left(x_{j}\right)=\left\{\begin{aligned}
f^{\prime}\left(x_{j}\right), & \text { if } x_{j} \text { is the left endpoint of an edge, } \\
-f^{\prime}\left(x_{j}\right), & \text { if } x_{j} \text { is the right endpoint of an edge. }
\end{aligned}\right.
$$

Associate either of two symbols, $\delta$ or $\delta^{\prime}$, to each vertex of the graph $\Gamma$. The graph thus obtained will be referred to as marked and denoted $\Gamma_{\delta}$. A vertex $V$ will be called a vertex of $\delta$ type, if $V$ is marked by the symbol $\delta$, and a vertex of $\delta^{\prime}$ type in the opposite case. Any marked graph $\Gamma_{\delta}$ determines the lineal

$\mathcal{D}\left(\Gamma_{\delta}\right):=\left\{\begin{array}{l|l}f \in W_{2}^{2}(\Gamma) & \begin{array}{l}f \text { is continuous at all } \\ \text { internal vertices of } \delta \text { type } \\ \partial_{n} f\left(x_{i}\right)=\partial_{n} f\left(x_{j}\right) \forall i, j: x_{i}, x_{j} \in V \text { at all } \\ \text { internal vertices } V \text { of } \delta^{\prime} \text { type }\end{array}\end{array}\right\}$.

Note that the latter definition imposes no restrictions on the functions from $\mathcal{D}\left(\Gamma_{\delta}\right)$ at boundary vertices of the graph, i.e., at vertices of valence 1. For reasons of convenience, we refer to all graph vertices of higher valence as internal vertices throughout.

We are now all set to define the Laplacian $A_{\vec{\alpha}}$ on the graph $\Gamma_{\delta}$ which is the operator of the negative second derivative on functions from $f \in \mathcal{D}\left(\Gamma_{\delta}\right)$ subject to the following additional matching conditions.

( $\delta)$ If $V_{k}$ is of $\delta$ type, then

$$
\sum_{x_{j} \in V_{k}} \partial_{n} f\left(x_{j}\right)=\alpha_{k} f\left(V_{k}\right)
$$

$\left(\delta^{\prime}\right)$ If $V_{k}$ is of $\delta^{\prime}$ type, then

$$
\sum_{x_{j} \in V_{k}} f\left(x_{j}\right)=-\alpha_{k} \partial_{n} f\left(V_{k}\right)
$$

Here $\vec{\alpha}=\left(\alpha_{1}, \alpha_{2}, \ldots \alpha_{N}\right)$ is a set of arbitrary real constants which we will refer to as coupling constants, whereas $f\left(V_{k}\right)$ and $\partial_{n} f\left(V_{k}\right)$ are welldefined on $\mathcal{D}\left(\Gamma_{\delta}\right)$ at vertices of $\delta$ and $\delta^{\prime}$ type, respectively.

Provided that all coupling constants $\alpha_{m}, m=1 \ldots N$, are real, the operator $A_{\vec{\alpha}}$ is self-adjoint in Hilbert space $L_{2}(\Gamma)$ [13, 18]. Throughout the present paper, we are going to consider this self-adjoint situation only, although it has to be noted that the approach developed can be used for the purpose of analysis of the general non-self-adjoint situation as well.

Clearly, the self-adjoint operator thus defined on a finite compact metric graph has purely discrete spectrum that accumulates to $+\infty$. 
In order to ascertain this, one only has to note that the operator considered is a finite-dimensional perturbation in the resolvent sense of the direct sum of Sturm-Liouville operators on individual edges.

Note that w.l.o.g. each edge $e_{j}$ of the graph $\Gamma$ can be considered to be an interval $\left[0, l_{j}\right]$, where $l_{j}=x_{2 j}-x_{2 j-1}, j=1 \ldots n$ is the length of the corresponding edge. Throughout the present paper we will therefore only consider this situation.

The analysis presented in the present paper is essentially based on the theory of boundary triples [15, 20, 21, 9] applied to the class of operators introduced above. Two fundamental concepts of this theory which we will recall below are the concepts of a boundary triple and of the Titchmarsh-Weyl generalized matrix-function. Assume that $A_{\text {min }}$ is a symmetric densely defined operator in Hilbert space $H$, and that its deficiency indices are equal. Put $A_{\max }:=A_{\text {min }}^{*}$.

Definition $1([15,20,9])$. Let $\Gamma_{0}, \Gamma_{1}$ be linear mappings of $\operatorname{dom}\left(A_{\max }\right)$ to $\mathcal{H}$ which is a separable Hilbert space. The triple $\left(\mathcal{H}, \Gamma_{0}, \Gamma_{1}\right)$ is called a boundary triple for the operator $A_{\max }$ if:

(1) for all $f, g \in \operatorname{dom}\left(A_{\max }\right)$

$$
\left(A_{\max } f, g\right)_{H}-\left(f, A_{\max } g\right)_{H}=\left(\Gamma_{1} f, \Gamma_{0} g\right)_{\mathcal{H}}-\left(\Gamma_{0} f, \Gamma_{1} g\right)_{\mathcal{H}} .
$$

(2) the mapping $\gamma$ defined as $f \longmapsto\left(\Gamma_{0} f ; \Gamma_{1} f\right), f \in \operatorname{dom}\left(A_{\max }\right)$ is surjective, i.e., for all $Y_{0}, Y_{1} \in \mathcal{H}$ there exists an element $y \in \operatorname{dom}\left(A_{\max }\right)$ such that $\Gamma_{0} y=Y_{0}, \Gamma_{1} y=Y_{1}$.

A non-trivial extension $A_{B}$ of the operator $A_{\text {min }}$ such that $A_{\text {min }} \subset$ $A_{B} \subset A_{\text {max }}$ is called almost solvable if there exists a boundary triple $\left(\mathcal{H}, \Gamma_{0}, \Gamma_{1}\right)$ for $A_{\max }$ and a bounded linear operator $B$ defined on $\mathcal{H}$ such that for every $f \in \operatorname{dom}\left(A_{\max }\right)$

$$
f \in \operatorname{dom}\left(A_{B}\right) \text { if and only if } \Gamma_{1} f=B \Gamma_{0} f .
$$

The operator-function $M(\lambda)$, defined by

$$
M(\lambda) \Gamma_{0} f_{\lambda}=\Gamma_{1} f_{\lambda}, f_{\lambda} \in \operatorname{ker}\left(A_{\max }-\lambda\right), \lambda \in \mathbb{C}_{ \pm},
$$

is called the Weyl-Titchmarsh $M$-function of the operator $A_{\max }$ w.r.t. the corresponding boundary triple.

The property of the Weyl-Titchmarsh $M$-function that makes it the tool of choice for the analysis of isospectral Laplacians on graphs can be formulated [30] in the following way: provided that $A_{B}$ is an almost solvable extension of a simple ${ }^{1}$ symmetric operator $A_{\min }, \lambda_{0} \in \rho\left(A_{B}\right)$ if

\footnotetext{
${ }^{1}$ I.e., there exists no reducing subspace $H_{0}$ such that the restriction $A_{\min } \mid H_{0}$ is a selfadjoint operator in $H_{0}$.
} 
and only if $(B-M(\lambda))^{-1}$ admits analytic continuation into the point $\lambda_{0}$.

In [12, we have obtained the following

Proposition 2.1 ([12]). Let $\Gamma_{\delta}$ be a marked compact metric graph. There exists a closed densely defined symmetric operator $A_{\min }$ and a boundary triple such that the operator $A_{\vec{\alpha}}$ is an almost solvable extension of $A_{\min }$, for which the parameterizing matrix $B$ is nothing but $\operatorname{diag}\left\{\alpha_{1}, \ldots, \alpha_{N}\right\}$, whereas the generalized Weyl-Titchmarsh $M$ function is a $N \times N$ matrix with matrix elements given by the following formula for a vertex $V_{k}$ of $\delta$ type: $m_{j k}(\lambda)=$

$$
\begin{cases}-\mu\left(\sum_{e_{t} \in E_{k}} \cot \mu l_{t}-\sum_{e_{t} \in E_{k}^{\prime}} \tan \mu l_{t}-\right. & \\ \left.-2 \sum_{e_{t} \in L_{k}} \tan \frac{\mu l_{t}}{2}\right), & j=k, \\ \mu \sum_{e_{t} \in C_{k j}} \frac{1}{\sin \mu l_{t}}, & j \neq k, V_{j} \text { is a vertex of } \\ -\sum_{e_{t} \in C_{k j}^{\prime}} \frac{1}{\cos \mu l_{t}}, & \delta \text { type adjacent to } V_{k}, \\ 0, & j \neq k, V_{j} \text { is a vertex of } \\ & \delta^{\prime} \text { type adjacent to } V_{k}, \\ & j \neq k, V_{j} \text { is a vertex } \\ & \text { not adjacent to } V_{k},\end{cases}
$$

and by the following formula for a vertex $V_{k}$ of $\delta^{\prime}$ type: $m_{j k}(\lambda)=$

$$
\begin{cases}-\frac{1}{\mu}\left(\sum_{e_{t} \in E_{k}} \cot \mu l_{t}-\sum_{e_{t} \in E_{k}^{\prime}} \tan \mu l_{t}+\right. & \\ \left.+2 \sum_{e_{t} \in L_{k}} \cot \frac{\mu l_{t}}{2}\right), & j=k, \\ -\sum_{e_{t} \in C_{k j}^{\prime}} \frac{1}{\cos \mu l_{t}}, & j \neq k, V_{j} \text { is a vertex of } \\ & \delta \text { type adjacent to } V_{k}, \\ -\frac{1}{\mu} \sum_{e_{t} \in C_{k j}} \frac{1}{\sin \mu l_{t}}, & j \neq k, V_{j} \text { is a vertex of } \\ 0, & \delta^{\prime} \text { type adjacent to } V_{k}, \\ & j \neq k, V_{j} \text { is a vertex } \\ & \text { not adjacent to } V_{k} .\end{cases}
$$

Here $\mu=\sqrt{\lambda}$ (the branch such that $\Im \mu \geq 0$ ), $l_{t}$ is the length of $e_{t}, L_{k}$ is the set of loops at the vertex $V_{k}, E_{k}$ is the set of graph edges incident to the vertex $V_{k}$ with both endpoints of the same type, $E_{k}^{\prime}$ is the set of graph edges incident to the vertex $V_{k}$ with endpoints of different types, $C_{k j}$ is the set of graph edges connecting vertices $V_{k}$ and $V_{j}$ of the same 
type, and finally, $C_{k j}^{\prime}$ is the set of graph edges connecting vertices $V_{k}$ and $V_{j}$ of different types.

This explicit description of the $M$-function w.r.t. the boundary triple that we are inclined to consider as the natural one has allowed us to prove necessary conditions of quantum graphs isospectrality. In order to achieve this goal, one has to look as the asymptotic expansion of the M-function as $\lambda \rightarrow-\infty$ along the real line, see details in [12].

\section{NECESSARY AND SUFFICIENT CONDITIONS OF ISOSPECTRALITY}

In the present Section we will obtain necessary and sufficient conditions of isospectrality provided that edge lengths of the graph are assumed to be rationally independent.

In what follows we will utilize the following three rather straightforward lemmata.

Lemma 3.1. Let $A_{\vec{\alpha}}$ be a Laplacian on the marked graph $\Gamma_{\delta}$. If all edge lengths of the graph $\Gamma_{\delta}$ are rationally independent, the function $\Pi(\lambda) \operatorname{det}(M(\lambda)-B)$ with $B=\operatorname{diag}\left\{\alpha_{1}, \ldots, \alpha_{N}\right\}$ and

$$
\Pi(\lambda)=\lambda^{\rho} \prod_{e_{j} \in \mathcal{C}} \frac{\sin l_{j} \sqrt{\lambda}}{\sqrt{\lambda}} \prod_{e_{j} \in \mathcal{C}^{\prime}} \cos l_{j} \sqrt{\lambda} \prod_{e_{j} \in \mathcal{L}} \cos l_{j} \frac{\sqrt{\lambda}}{2} \prod_{e_{j} \in \mathcal{L}^{\prime}} \frac{\sin l_{j} \frac{\sqrt{\lambda}}{2}}{\sqrt{\lambda}}
$$

is an entire function of exponential type and order not greater than $1 / 2$ in $\mathbb{C}$, if $\rho$ is the number of vertices of $\delta^{\prime}$ type connected in $\Gamma_{\delta}$ with at least one vertex of this same type (either via an edge or a loop).

Here $\mathcal{C}\left(\mathcal{C}^{\prime}\right)$ is the set of edges connecting vertices of same type (of different types, respectively); $\mathcal{L}\left(\mathcal{L}^{\prime}\right)$ is the set of loops attached to vertices of type $\delta$ (of type $\delta^{\prime}$, respectively).

Proof. Due to Proposition 2.1 it suffices to prove that the function in question has no poles in $\mathbb{C}$. Then the fact that it has claimed exponential type and order follows immediately from [27] since it can be represented as a fraction of two entire functions of this same order.

From Proposition 2.1 we deduce that poles could only be located at zeroes of the function $\Pi(\lambda)$. Consider the possibilities.

(i) Let the edge $e_{t}$ of length $l_{t}$ connect vertices $V_{k}$ and $V_{j}$ both of type $\delta$. Then by (1) the entries $m_{k k}, m_{j j}$ of $M(\lambda)$ contain the summand $-\mu \cot \mu l_{t}$ corresponding to $e_{t}$, whereas the entries $m_{k j}, m_{j k}$ contain $\mu \csc \mu l_{t}$. Moreover, no other entries of $M(\lambda)$ contain trigonometric functions of the same argument. Add the $j$ th row multiplied by $\cos \mu l_{t}$ to the $k$ th one. Then in $m_{k j}$ the term $\mu \csc \mu l_{t}$ admits the form $\mu \sin \mu l_{t}$, whereas the term $-\mu \cot \mu l_{t}$ in $m_{k k}$ cancels out. Factoring out $\frac{1}{\sin \mu l_{t}}$ 
from the $j$ th row we see that the function $\Pi(\lambda) \operatorname{det}(M(\lambda)-B)$ has no poles associated with zeroes of $\sin \mu l_{t}$.

(ii) If the edge $e_{t}$ of length $l_{t}$ connects vertices $V_{k}$ and $V_{j}$ both of type $\delta^{\prime}$, the same argument as in (i) above applies.

(iii) Let the edge $e_{t}$ of length $l_{t}$ connect vertices $V_{k}$ and $V_{j}$ having $\delta$ and $\delta^{\prime}$ types, respectively. In this case by (2) $m_{k k}$ contains the summand $\mu \tan \mu l_{t}$ and $m_{j j}$ contains $\frac{1}{\mu} \tan \mu l_{t}$, corresponding to this edge, whereas the entries $m_{k j}, m_{j k}$ contain the term $-\sec \mu l_{t}$. Moreover, no other matrix elements of $M(\lambda)$ contain trigonometric functions of the same argument. Add the $j$ th row multiplied by $\mu \cos \mu l_{t}$ to the $k$ th one. Then in $m_{k j}$ the term $-\sec \mu l_{t}$ admits the form $-\cos \mu l_{t}$, whereas the term $\mu \tan \mu l_{t}$ in $m_{k k}$ cancels out. Factoring out $\frac{1}{\cos \mu l_{t}}$ from the $j$ th row we see that the function $\Pi(\lambda) \operatorname{det}(M(\lambda)-B)$ has no poles associated with zeroes of $\cos \mu l_{t}$.

(iv) The case of loops attached to vertices of either $\delta$ or $\delta^{\prime}$ type is of course trivial, see (1) and (2).

Finally, as it is easily seen, the factor $\lambda^{\rho}$ ensures that the function in question has no pole at zero, cf. Theorem 3.4 below.

Lemma 3.2. Assume that $A_{\vec{\alpha}}$ and $A_{\overrightarrow{\tilde{\alpha}}}$ are two Laplacians on the graph $\Gamma_{\delta}$. Assume that all edge lengths are rationally independent. These operators are isospectral if and only if the numbers of zero coupling constants at vertices of $\delta^{\prime}$ type are equal in $\vec{\alpha}$ and $\overrightarrow{\tilde{\alpha}}$ and

$$
\Phi(\vec{\alpha}) \Pi(\lambda) \operatorname{det}(M(\lambda)-B) \equiv \Phi(\overrightarrow{\tilde{\alpha}}) \Pi(\lambda) \operatorname{det}(M(\lambda)-\tilde{B}) \text { for all } \lambda \in \mathbb{C},
$$

where $B=\operatorname{diag}\left\{\alpha_{1}, \ldots, \alpha_{N}\right\}$ and $\tilde{B}=\operatorname{diag}\left\{\tilde{\alpha}_{1}, \ldots, \tilde{\alpha}_{N}\right\}$;

$$
\Phi(\vec{\alpha}):=\prod_{V_{j} \text { of } \delta^{\prime} \text { type: } \alpha_{j} \neq 0} \frac{\operatorname{deg} V_{j}}{\alpha_{j}} .
$$

Proof. The "only if" part follows from Lemma 3.1 and Proposition 2.1 by Hadamard theorem, see [12] for details.

Consider the "if" part. In the case when $A_{\min }$ is simple (i.e., contains no reducing self-adjoint "parts") the proof is trivially based on the fact mentioned above that "zeroes" of $M(\lambda)-B$ are located exactly at the points of spectrum of the operator $A_{B} \equiv A_{\vec{\alpha}}$ taking into account that $M$-function is an R-function in both half-planes of the complex plane with almost everywhere Hermitian values on $\mathbb{R}$, see [9].

By [1] the condition of rational independence of edge lengths in fact guarantees this simplicity whenever the graph contains no loops.

If the graph contains loops, we are sure to have parts of the spectrum of $A_{B}$ "invisible" to the function $M(\lambda)-B$, but due to [10, 1] the 
"invisible" part of the spectrum coincides with the point spectrum of $A_{\text {min }}$, which is identical for $A_{B}$ and $A_{\tilde{B}}$.

We remark that the condition that the numbers of zero coupling constants at vertices of $\delta^{\prime}$ type are equal in $\vec{\alpha}$ and $\overrightarrow{\tilde{\alpha}}$ is not needed to prove the "if" part of the latter Lemma since by an argument of [12] it already follows from $\operatorname{det}(M(\lambda)-B) / \operatorname{det}(M(\lambda)-\tilde{B})=$ const. This condition is nonetheless vital for correctness of (5) and (44).

Assume that $M$ is a symmetric $N \times N$ matrix with matrix elements $m_{i j}$. Let the weighted oriented graph $\Gamma(M)$ of exactly $N$ vertices be constructed in the following way. For every $i=1, \ldots, N$ attach a loop carrying the weight $m_{i i}$ to the $i$ th vertex. For all $i \neq j$ such that the matrix element $m_{i j}$ is non-zero, draw two edges (one in each direction) connecting the $i$ th and $j$ th vertices, each carrying the weight $m_{i j}$.

The following Lemma holds.

Lemma 3.3. Let $M$ be a symmetric $N \times N$ matrix with matrix elements $m_{i j}$; let $\Gamma(M)$ be the weighted oriented graph associated with $M$ as described above.

Let $\mathcal{G}$ be the set of all spanning (i.e., containing all vertices of $\Gamma(M)$ ) subgraphs of $\Gamma(M)$ which are unions of non-intersecting loops and properly oriented cycles.

Then one has the following formula for $\operatorname{det} M$ :

$$
\operatorname{det} M=\sum_{G \in \mathcal{G}}(-1)^{N(G)} \prod_{e \in E(G)} w(e)
$$

where the sum is taken over all subgraphs $G \in \mathcal{G} ; E(G)$ is the set of edges of $G, w(e)$ is the weight of the edge $e$ and finally $N(G)$ is the number of cycles containing even number of edges (even cycles) in $G$.

Remark 1. This Lemma is probably well known in some similar form. We have picked up the idea in [32], where it is used in the framework of spectral theory of discrete Laplace operators (and then $\sum_{j=1}^{N} m_{i j}=$ $0 \forall i$, which simplifies the result). For the sake of completeness, we provide the proof below.

Proof. By the definition of determinant,

$$
\operatorname{det} M=\sum_{\left\{a_{1}, \ldots, a_{n}\right\}}(-1)^{\tau\left(a_{1}, a_{2}, \ldots, a_{n}\right)} m_{1 a_{1}} \ldots m_{n a_{n}},
$$

where $\left\{a_{1}, \ldots, a_{n}\right\}$ is a permutation of the set $\{1, \ldots, n\}$ and $\tau\left\{a_{1}, a_{2}, \ldots, a_{n}\right\}$ is its parity. Consider an arbitrary nonzero monomial $m_{1 a_{1}} \ldots m_{n a_{n}}$ of this sum. All factors of the form $m_{i i}$ correspond to loops of $\Gamma(M)$. Let 
exactly $k$ factors in this monomial be diagonal elements; consider the remaining part of it, $m_{i_{1} a_{i_{1}}} \ldots m_{i_{N-k} a_{i_{N-k}}}$. The permutation $\left\{a_{i_{1}}, \ldots, a_{i_{N-k}}\right\}$ of the set $\left\{i_{1}, \ldots, i_{N-k}\right\}$ can obviously be decomposed into the product of independent permutations, each of which corresponds to a certain simple cycle of $\Gamma(M)$. Moreover, these cycles do not have common vertices.

Therefore, the monomial $m_{1 a_{1}} \ldots m_{n a_{n}}$ is nothing but the product of edge weights of a spanning subgraph $G \in \Gamma(M)$ which is a collection of loops and simple cycles. On the other hand, it is easily seen that for any subgraph of this type there exists a corresponding monomial in $\operatorname{det} M$.

As for the sign, $(-1)^{N(G)}=\tau\left\{a_{1}, a_{2}, \ldots, a_{n}\right\}$ since the parity of a permutation is exactly the number of even permutations under the decomposition into the product of independent factors.

We remark that if some matrix elements $m_{i j}$ are decomposable into sums, $m_{j i}=m_{i j}=\mu_{i j}+\nu_{i j}$, it is natural to modify the construction of the graph $\Gamma(M)$ in a way such that this graph: (i) has two loops with weights $\mu_{i i}$ and $\nu_{i i}$ attached to the vertex $V_{i}$ in the case of $i=j$; (ii) has two edges carrying weights $\mu_{i j}$ and $\nu_{i j}$, respectively, directed from the vertex $V_{i}$ to the vertex $V_{j}$ and two edges of same weights in the opposite direction. In this situation, the proof just given applies verbatim.

An inspection of (11) and (2) shows how this idea can be utilized to control the determinant of $M(\lambda)-B$. Note that this matrix bears close resemblance to the adjacency matrix of the graph $\Gamma_{\delta}$ in the sense that (i) it is symmetric and (ii) any non-diagonal matrix element of it is zero iff the corresponding two vertices are disconnected in $\Gamma_{\delta}$. We start by describing the corresponding modification procedure for the graph $\Gamma_{\delta}$, which is to yield the graph $\Gamma(M(\lambda)-B)$ in terms of Lemma 3.3.

Modification of the graph $\Gamma_{\delta} .1$. The set of vertices of $\Gamma_{\delta}$ remains unchanged.

2. Every edge $e$ of length $l$ which is not a loop is doubled (with both instances assigned opposite directions); the same weight $w(e)$ is assigned to both instances:

$$
\omega(e)= \begin{cases}\mu \csc \mu l, & \text { if } e \text { connects vertices of } \delta \text { type; } \\ \frac{1}{\mu} \csc \mu l, & \text { if } e \text { connects vertices of } \delta^{\prime} \text { type; } \\ -\sec \mu l, & \text { if } e \text { connects vertices of different types. }\end{cases}
$$


3. Every edge $e$ of length $l$ connecting a vertex $V$ to any other vertex $W$ induces a loop $\mathcal{O}$ attached to the vertex $V$ carrying the weight $w(\mathcal{O})$ : $w(\mathcal{O})= \begin{cases}-\mu \cot \mu l, & \text { if } V, W \text { are both of } \delta \text { type; } \\ -\frac{1}{\mu} \cot \mu l, & \text { if } V, W \text { are both of } \delta^{\prime} \text { type; } \\ \mu \tan \mu l, & \text { if } V \text { is a vertex of } \delta \text { type and } W \text { is of } \delta^{\prime} \text { type; } \\ \frac{1}{\mu} \tan \mu l, & \text { if } V \text { is a vertex of } \delta^{\prime} \text { type and } W \text { is of } \delta \text { type. }\end{cases}$

3. Each loop $e$ of length $l$ attached to a vertex $V$ to be found in the graph $\Gamma_{\delta}$ is assigned the weight $w(e)$ :

$$
w(e)= \begin{cases}2 \mu \tan \frac{\mu l}{2}, & \text { if } V \text { is of } \delta \text { type; } \\ -\frac{2}{\mu} \cot \frac{\mu l}{2}, & \text { if } V \text { is of } \delta^{\prime} \text { type. }\end{cases}
$$

4. Finally, attach a loop of weight $-\alpha_{V}$ to every vertex $V$, where $\alpha_{V}$ is the coupling constant pertaining to the vertex $V$. These loops will henceforth be treated specially; we will refer to them as alpha-loops.

We will denote the graph $\Gamma_{\delta}$ so modified by the symbol $\Gamma_{\delta}^{(\text {mod })}$.

Treatment of subgraphs for $\Gamma_{\delta}^{(\text {mod })}$. Let as in Lemma $3.3 \mathcal{G}$ be the set of all spanning subgraphs of $\Gamma_{\delta}^{(\bmod )}$ which are unions of non-intersecting loops and properly oriented cycles.

The named Lemma then gives for $\operatorname{det}(M(\lambda)-B)$ :

$$
\operatorname{det}(M(\lambda)-B)=\sum_{G \in \mathcal{G}}(-1)^{N(G)} \prod_{e \in E(G)} w(e) .
$$

Each subgraph $G \in \mathcal{G}$ can be uniquely decomposed into a graph which is a collection of alpha-loops $G_{\alpha}$ and the remainder $G_{\bar{\alpha}}$ :

$$
G=G_{\alpha}+G_{\bar{\alpha}}
$$

When $G$ is the union of all alpha-loops of $\Gamma_{\delta}^{(\bmod )}$, for reasons of convenience we will write $G_{\bar{\alpha}}:=\mathbb{O}$ - the empty subgraph, to which we ascribe the weight $1, w(\mathbb{O})=1$. The same convention will be applied in the situation when $G$ contains no alpha-loops, i.e., $G=G_{\bar{\alpha}}$; in this case, we will put $G_{\alpha}:=\mathbb{O}$.

Let the set $\mathcal{G}_{\alpha}$ be the set of all $G_{\bar{\alpha}}$ including $\mathbb{O}$ as $G$ spans $\mathcal{G}$ :

$$
\mathcal{G}_{\bar{\alpha}}=\left\{G_{\bar{\alpha}} \mid G \in \mathcal{G}\right\} \text {. }
$$

If one introduces the natural notation $w(G):=\prod_{e \in E(G)} w(e)$ and takes into account that clearly $w(G)=w\left(G_{\alpha}\right) w\left(G_{\bar{\alpha}}\right)$, one immediately gets by Lemma 3.3

$$
\operatorname{det}(M(\lambda)-B)=\sum_{G \in \mathcal{G}_{\bar{\alpha}}}(-1)^{N(G)} w\left(G_{\alpha}\right) w(G),
$$


where $G_{\alpha}$ is a collection of alpha-loops needed to build $G$ up to a spanning subgraph of $\mathcal{G}$; obviously, $G_{\alpha}$ is defined uniquely by $G$. Moreover, $w\left(G_{\alpha}\right)$ is always a product (up to the sign) of those coupling constants $\alpha_{i}$ which appear as the weights in $G_{\alpha}$.

Had no further reductions in the set $\mathcal{G}_{\bar{\alpha}}$ been possible, we would have been all done by now. Yet, clearly $\mathcal{G}_{\bar{\alpha}}$ always contains exactly two graphs of the form $G_{1}=G_{0}+G_{f}$ and $G_{2}=G_{0}+G_{e}$, where $G_{f}$ is a properly directed loop of exactly two vertices, say $V$ and $W$, connected in $\Gamma_{\delta}$ by an edge of length $l$, with equal weights $w$ on both edges, whereas $G_{e}$ is a graph of two disjoint loops of weights $w^{\prime}=$ $w^{\prime}(w), w^{\prime \prime}=w^{\prime \prime}(w)$ attached to $V$ and $W$, respectively. Here $w^{\prime}(w)$ and $w^{\prime \prime}(w)$ can be either: (i) $-\mu \cot \mu l,-\mu \cot \mu l$, if both $V$ and $W$ are of $\delta$ type, or (ii) $-\mu^{-1} \cot \mu l,-\mu^{-1} \cot \mu l$, if both $V$ and $W$ are of $\delta^{\prime}$ type, or, finally, (iii) $\mu \tan \mu l, \mu^{-1} \tan \mu l$, if $V$ and $W$ are of different types.

Consider the three named possibilities.

(i) If both $V$ and $W$ are of $\delta$ type, one gets $w\left(G_{f}\right)=\mu^{2} / \sin ^{2}(\mu l)$ and $w\left(G_{e}\right)=\mu^{2} \cot ^{2}(\mu l)$. By the main trigonometric identity one then has:

$$
(-1)^{N\left(G_{1}\right)} w\left(G_{1}\right)+(-1)^{N\left(G_{2}\right)} w\left(G_{2}\right)=(-1)^{N\left(G_{1}\right)} \mu^{2} w\left(G_{0}\right) .
$$

(ii) If both $V$ and $W$ are of type $\delta^{\prime}$, one has $w\left(G_{f}\right)=\csc ^{2}(\mu l) / \mu^{2}$ and $w\left(G_{e}\right)=\cot ^{2}(\mu l) / \mu^{2}$ to the same effect.

(iii) Finally, if $V$ and $W$ are of different types, one gets the possibility of $w\left(G_{f}\right)=\sec ^{2}(\mu l)$ and $w\left(G_{e}\right)=\tan ^{2}(\mu l)$ leading to the cancellation of the same type.

Surely $G_{0}$ itself might be decomposable into either $G_{0}=G_{0}^{\prime}+G_{f}^{\prime}$ or $G_{0}=G_{0}^{\prime}+G_{e}^{\prime}$, but if, say, the former of the two decompositions holds, the subgraph $\tilde{G}_{0}=G_{0}^{\prime}+G_{e}^{\prime} \in \mathcal{G}_{\bar{\alpha}}$. In turn, the cancellation procedure for $\tilde{G}_{0}+G_{f}$ and $\tilde{G}_{0}+G_{e}$ as described above is applicable. Then one arrives at the possibility of the corresponding cancellation procedure between $G_{0}$ and $\tilde{G}_{0}$. The same argument applies to the second named possibility.

Therefore, the cancellation described above can be applicable repeatedly. Lemma 3.1 easily implies that it results in no subgraph weights which involve squares of trigonometric functions; on the other hand, no higher powers are possible due to the condition that edge lengths of $\Gamma_{\delta}$ are rationally independent.

The above argument gives ground to one further step in modification of $\mathcal{G}_{\bar{\alpha}}$. 
Definition 2. We define $\hat{\mathfrak{G}}$ as the set $\mathcal{G}_{\bar{\alpha}}$, to each graph $G$ of which the following procedure is applied.

I. Assume that $G \in \mathcal{G}_{\bar{\alpha}}$ contains two vertices, connected in $\Gamma_{\delta}$ by an edge of length $l$, to which the loops with weights of either $-\mu \cot \mu l$, $-\mu \cot \mu l$, or $\mu \tan \mu l, \mu^{-1} \tan \mu l$, or $-\mu^{-1} \cot \mu l,-\mu^{-1} \cot \mu l$ are attached, respectively. In this case, eliminate this subgraph $G$ altogether.

II. Assuming that $G \in \mathcal{G}_{\bar{\alpha}}$ contains a directed cycle of exactly two vertices with equal weights $w$ on both edges, replace the weights $w$ by: (i) $\mu$, if both vertices are of $\delta$ type; (ii) 1 , if they are of different types and (iii) $1 / \mu$, if both vertices are of type $\delta^{\prime}$.

Finally, introduce the following equivalence relation. Put $G_{1} \sim G_{2}$, if $w\left(G_{1}\right)=w\left(G_{2}\right)$, and define the factored set

$$
\mathfrak{G}:=\hat{\mathfrak{G}} / \sim .
$$

We have thus arrived at the following

Theorem 3.4. Assume that $A_{\vec{\alpha}}$ is a Laplacian on the graph $\Gamma_{\delta}$ with coupling constants $\left\{\alpha_{1}, \ldots, \alpha_{N}\right\}$. Let $M(\lambda)$ be its Weyl-Titchmarsh $M$ function provided by Proposition [2.1, and let $B=\operatorname{diag}\left\{\alpha_{1}, \ldots, \alpha_{N}\right\}$. Then

$$
\operatorname{det}(M(\lambda)-B)=\sum_{\gamma \in \mathfrak{G}} f_{\gamma}(\vec{\alpha}) w(\gamma)
$$

where $\mathfrak{G}$ is defined by (6), $\gamma$ is treated as an equivalence class belonging to $\mathfrak{G}$ with the natural definition of $w(\gamma)$ as the weight of any of subgraphs $G$ of this equivalence class and

$$
f_{\gamma}(\vec{\alpha})=\sum_{G \in \gamma}(-1)^{N(G)} w\left(G_{\alpha}\right),
$$

$G_{\alpha}$ as above being a collection of alpha-loops needed to build $G$ up to a spanning subgraph of $\mathcal{G}$, uniquely determined by $G$.

Remark 2. One can easily ascertain that in the situation of all vertices of $\Gamma_{\delta}$ being of the same type (i.e., either $\delta$ or $\delta^{\prime}$ ) the function $f_{\gamma}(\vec{\alpha})$ for each $\gamma \in \mathfrak{G}$ is homogeneous w.r.t. its argument. It is in particular linear in $\left\{\alpha_{i}\right\}_{i=1}^{N}$ for any class $\gamma$ such that $G_{\alpha}, \forall G \in \gamma$ is a single alpha-loop.

The result of Theorem 3.4 allows to reformulate Lemma 3.2 in the following explicit form.

Theorem 3.5. Assume that $A_{\vec{\alpha}}$ and $A_{\overrightarrow{\widetilde{\alpha}}}$ are two Laplacians on the graph $\Gamma_{\delta}$. Assume that all edge lengths are rationally independent. 
These operators are isospectral if and only if the numbers of zero coupling constants at vertices of $\delta^{\prime}$ type are equal and

$$
\Phi(\vec{\alpha}) f_{\gamma}(\vec{\alpha})=\Phi(\overrightarrow{\tilde{\alpha}}) f_{\gamma}(\overrightarrow{\tilde{\alpha}}), \quad \forall \gamma \in \mathfrak{G},
$$

where $f_{\gamma}$ and $\mathfrak{G}$ are as in Theorem 3.4; the function $\Phi$ is defined in Lemma 3.2.

Proof. The "only if" part follows immediately from Lemma 3.2, taking into account linear independence of all functions of the form $\Pi(\lambda) w(\gamma)$, provided for by the condition of rational independence of edge lengths.

The "if" part is trivial by Theorem 3.4 and Lemma 3.2 .

One has to admit that the result of Theorem 3.2 at the first glance does not seem to be suitable for applications. Indeed, for a given graph $\Gamma_{\delta}$, even having explicitly calculated functions $f_{\gamma}$, one faces the necessity to consider all possibilities for $\Phi(\vec{\alpha})$ and $\Phi(\overrightarrow{\tilde{\alpha}})$ in turn. These are to include all admissible configurations of zero coupling constants at $\delta^{\prime}$ type vertices independently for $\vec{\alpha}$ and $\overrightarrow{\tilde{\alpha}}$. Each of these configurations on the face of it gives rise to a different set of necessary and sufficient conditions of isospectrality.

However, matters do simplify to a great extent provided that one faces the situation of $\Phi(\vec{\alpha})=\Phi(\overrightarrow{\tilde{\alpha}})$ under the assumption of isospectrality. Indeed, not only the awkward first factors then disappear from conditions (8), but functions $f_{\gamma}$ also admit further simplification. In order to ascertain this, note that by construction some of functions $f_{\gamma}$ may contain constant summands. These clearly come from $\gamma \in \mathfrak{G}$ containing at least one $G \in \mathcal{G}$ such that $G_{\alpha}=\mathbb{O}$. Under the additional assumption $\Phi(\vec{\alpha})=\Phi(\overrightarrow{\tilde{\alpha}})$ these constant terms can clearly be dropped altogether. For any $\gamma$ decompose $f_{\gamma}(\vec{\alpha})=c_{\gamma}+g_{\gamma}(\vec{\alpha})$, where $c_{\gamma}$ is the term in $f_{\gamma}$ independent of $\vec{\alpha}$. Then one has

Corollary 3.6. Assume that $A_{\vec{\alpha}}$ and $A_{\overrightarrow{\widetilde{\alpha}}}$ are two Laplacians on the graph $\Gamma_{\delta}$. Assume that all edge lengths are rationally independent. Let the condition of isospectrality yield

$$
\Phi(\vec{\alpha})=\Phi(\overrightarrow{\tilde{\alpha}})
$$

Then the operators are isospectral if and only if the numbers of zero coupling constants at vertices of $\delta^{\prime}$ type are equal and

$$
g_{\gamma}(\vec{\alpha})=g_{\gamma}(\overrightarrow{\tilde{\alpha}}), \quad \forall \gamma \in \mathfrak{G} .
$$

It turns out that in general (9) cannot be guaranteed (see Example 1 below) even in the situation of rationally independent edge lengths. Nevertheless one might argue that almost all graphs $\Gamma_{\delta}$ have this property. This claim is in fact a corollary of what is to follow. 
Note first that equality (9) under the assumption of isospectrality is in fact guaranteed [12] in the case when all graph vertices are of the same type (trivially in the $\delta$ case).

In the general situation, first assume that the graph $\Gamma_{\delta}$ is not a tree. Then one is sure to have a subgraph $G \in \mathcal{G}$ (cf. Lemma 3.3) with $G_{\alpha}=\mathbb{O}$ such that for no graph $\tilde{G} \in \mathcal{G}$ with $G_{\alpha} \neq \mathbb{O}$ the equality $w\left(\tilde{G}_{\bar{\alpha}}\right)=w(G)$ holds.

This subgraph is nothing but a set of $N$ regular loops (i.e., non-alpha loops).

The proof of this is a straightforward application of the following simple

Lemma 3.7. Assume that $\Gamma$ is a graph with each edge assigned a different weight $l_{j}$. Call a marking of graph vertices admissible if any vertex is allowed to be marked by either of the weights $l_{j}$ of the edges incident to it and no edge has both endpoints marked by its weight at the same time, unless this edge is a loop. Then

(i) if the graph is not a tree, there exists an admissible marking of vertices such that no proper subgraph $G \subsetneq \Gamma$ has an admissible marking with the same set of marks;

(ii) if the graph is a tree, there exists no admissible marking. In this situation, the maximal number of different weights appearing in a marking equals $n=N-1$; this "maximal" marking has exactly one edge having both endpoints marked with its weight. For any edge of the graph, a maximal marking is constructed uniquely such that this particular edge and this edge only has both endpoints marked by the same weight.

Proof. We start with (ii). Take any vertex $V$ of the tree $\Gamma$ as the root. Mark any vertex $V^{\prime}$ adjacent to it with the weight of the edge connecting it to $V$. Repeat this step for every $V^{\prime}$ in the rôle of $V$ and carry on until the graph is over. Now pick any of the edges incident to the root $V$ and mark $V$ with its weight. The claim follows.

Consider (i). Pick a vertex $V$ belonging to at least one cycle of the graph or a vertex $V$ with a loop attached to it provided that $\Gamma$ contains no cycles. Take a spanning tree $T$ such that at least one edge $e$ of $\Gamma$ incident to $V$ is missing from it. Mark all vertices but $V$ as above, then mark the vertex $V$ with the weight of $e$.

The second part of the claim follows from the fact that any proper subgraph of $\Gamma$ has at least one vertex less than $\Gamma$. By construction of the marking, there are no repeating weights in it, thus any proper subgraph will have at least one weight less. 
Applying Theorem 3.5 to a pair of graph Laplacians on a non-tree graph $\Gamma_{\delta}$ which are assumed to be isospectral one then immediately arrives at the identity $\Phi(\vec{\alpha}) \equiv \Phi(\overrightarrow{\tilde{\alpha}})$.

We now pass over to the surprisingly much more involved analysis of the situation when $\Gamma_{\delta}$ is a tree.

Assume again that we have two isospectral Laplacians on the graph $\Gamma_{\delta}$. Then by [12], one has the set equality $S(\vec{\alpha})=S(\overrightarrow{\tilde{\alpha}})$, where $S(\vec{\alpha}):=$ $\left\{\sigma_{1}, \ldots, \sigma_{N}\right\}$ with

$$
\sigma_{i}:= \begin{cases}-\alpha_{i} / \operatorname{deg} V_{i} & \text { if } V_{i} \text { is of type } \delta \\ \operatorname{deg} V_{i} / \alpha_{i} & \text { if } V_{i} \text { is of type } \delta^{\prime} \text { and } \alpha_{i} \neq 0 \\ 0 & \text { otherwise }\end{cases}
$$

for any $i=1, \ldots, N$.

It follows that

$$
\prod_{\delta}^{\prime}\left(-\alpha_{i} / \operatorname{deg} V_{i}\right) \prod_{\delta^{\prime}}^{\prime}\left(\operatorname{deg} V_{i} / \alpha_{i}\right)=\prod_{\delta}^{\prime}\left(-\tilde{\alpha}_{i} / \operatorname{deg} V_{i}\right) \prod_{\delta^{\prime}}^{\prime}\left(\operatorname{deg} V_{i} / \tilde{\alpha}_{i}\right)
$$

where the symbol $\prod^{\prime}$ means that the product is taken over non-zero values of $\alpha_{i}$; the products are taken over all vertices of types indicated by the subscript.

Consider the case when there are no zero coupling constants in $\vec{\alpha}$ (and hence, in $\overrightarrow{\tilde{\alpha}}$ ). The reduction of the general situation to the named one will be discussed in the next Section.

The identity (12) implies that the only way of having $\Phi(\vec{\alpha}) \neq \Phi(\overrightarrow{\tilde{\alpha}})$ under the assumption of isospectrality is to have a number of values $\sigma_{i}$ redistributed somehow between graph vertices of different types.

We will henceforth assume that every edge of the graph $\Gamma_{\delta}$ is of "mixed" type (i.e., its endpoints have different types) and consider three different cases separately. The general case can be reduced to this one, see Section 4 for details.

A vertex of valence 2. Assume w.l.o.g. that $V$ is a $\delta$ type vertex of valence 2 (the case of $\delta^{\prime}$ type can be treated along the same lines). Consider the following special choice of $G \in \mathcal{G}$ : take an alpha-loop at each graph vertex of $\delta^{\prime}$ type and the alpha-loop at the vertex $V$. Take a regular loop at each other vertex. In order to fix $G$ uniquely, we use the marking provided by Lemma 3.7 for the graph $\Gamma$ under the assumption that the vertex $V$ is taken as a root; clearly, since at $V$ subgraph $G$ has an alpha-loop, any other $\delta$ type vertex will then contribute a loop of a unique weight to $G$. 
Consider all subgraphs $\tilde{G} \in \mathcal{G}$ which are equivalent to $G$ in the following sense: $\tilde{G}_{\bar{\alpha}} \sim G_{\bar{\alpha}}$ under the usual decomposition $G=G_{\alpha}+G_{\bar{\alpha}}$ and $\tilde{G}=\tilde{G}_{\alpha}+\tilde{G}_{\bar{\alpha}}$. This set of subgraphs $\tilde{G}$ can be explicitly described. Note that any edge $e$ incident to the vertex $V$ contributes alpha-loops associated to both its endpoints to $G$. For a fixed $e$ replace now these two alpha-loops by the corresponding directed cycle of two vertices. Clearly, the subgraph $\tilde{G}_{e}$ thus constructed is equivalent to $G$ since the weight of a two-cycle is equal to 1 .

There are no other subgraphs $\tilde{G} \in \mathcal{G}$ equivalent to $G$. To prove this claim, one has to note that any subgraph equivalent to $G$ must not only have the same collection of tangents $\tan l_{j} \mu$, but also the same combined power of $\mu$ in its weight (cf. Proposition 2.1). Since all graph vertices of $\delta$ type carry loops with weights of the form $\mu \tan l_{j} \mu$, whereas vertices of $\delta^{\prime}$ type have loops with $\mu^{-1} \tan l_{j} \mu$ weights, $G$ by construction has the factor $\mu^{\#(\delta)-1}$ in its weight, where $\#(\delta)$ is the number of $\delta$ type vertices of $\Gamma$. It follows that any subgraph equivalent to $G$ must have exactly the same number of loops coming from $\delta$ type vertices, i.e., $\#(\delta)-1$. Since the selection of edge lengths appearing in tangents is fixed by the choice of $G$ and at the same time there are no edges of $\delta-\delta$ and $\delta^{\prime}-\delta^{\prime}$ type in $\Gamma$ by assumption, this implies: away from $V$ and the vertices adjacent to it $\tilde{G}$ should be exactly the same as $G$. Trivial combinatorics now completes the proof.

By Theorem 3.5 one then easily obtains the following formula linking together coupling constants of two isospectral Laplacians:

$$
\alpha_{V}-\sum_{V^{\prime} \text { adjacent to } V} \frac{1}{\alpha_{V^{\prime}}}=\tilde{\alpha}_{V}-\sum_{V^{\prime} \text { adjacent to } V} \frac{1}{\tilde{\alpha}_{V^{\prime}}},
$$

where $\alpha_{V}$ is the coupling constant at the vertex $V$. We will henceforth refer to this condition as to the balancing condition at the vertex $V$.

One can go one step further. Consider any of the two subgraphs $\tilde{G}_{e}$ equivalent to $G$ described above. Modify $\tilde{G}_{e}$ in the following way. Replace the directed two-cycle corresponding to the edge $e$ of $\Gamma$ by two regular loops in a way such that there are no repeating weights in the subgraph loops still. There clearly exists a unique way of doing so. Denote the subgraph thus constructed by $\hat{G}_{e}$. The weight of $\hat{G}_{e}$ contains exactly the same power of $\mu$ as that of $\tilde{G}_{e}$ and $G$, differing from them in two additional tangents. By inspection, the weights of $\hat{G}_{e}$ for both edges $e$ incident to the vertex $V$ contain exactly the same collection of tangents. An argument similar to the one presented above shows that there are no further subgraphs equivalent to $\hat{G}_{e}$. Theorem 3.2 applied to the class of equivalence $\gamma$ such that $\hat{G}_{e} \in \gamma$ now implies 
that

$$
\sum_{V^{\prime} \text { adjacent to } V} \frac{1}{\alpha_{V^{\prime}}}=\sum_{V^{\prime} \text { adjacent to } V} \frac{1}{\tilde{\alpha}_{V^{\prime}}},
$$

and thus $\alpha_{V}=\tilde{\alpha}_{V}$ by the balancing condition (13) for the vertex $V$ considered.

$A$ vertex of valence 1 . Now consider the case of a boundary vertex $V$ of $\delta$ type (the case of $\delta^{\prime}$ type is treated in a similar way).

The balancing condition (13) is obtained as in the case of $\operatorname{deg} V=2$ discussed above, verbatim. The difference with the latter case is that here one cannot take the second step described above. This follows from the fact that for a boundary vertex there exists no possibility to construct $\hat{G}_{e}$ : any attempt at doing so results in a subgraph having two loops with exactly the same weights at adjacent vertices. On the other hand, such subgraphs have to be eliminated by Definition 2 and thus do not belong to $\mathfrak{G}$.

A vertex of valence greater than 2. Finally assume that $V$ is a $\delta$ type (w.l.o.g.: the case of $\delta^{\prime}$ is treated similarly) vertex of higher valence.

Proceeding as above, one gets the balancing condition (13).

As in the case of $\operatorname{deg} V=2$, one can go one step further. In order to do so, note that in the case of higher valences there are $C_{d}^{2}$ non-directed 2-paths through the vertex $V$, where $d=\operatorname{deg} V$. Each of these paths gives rise to a pair of equivalent subgraphs $\hat{G}_{e}, \hat{G}_{e^{\prime}}$, where $\left(e, e^{\prime}\right)$ is the corresponding path through $V$. Moreover, it is easily seen that for different paths $e, e^{\prime}$ one arrives at different equivalence classes $\gamma \in \mathfrak{G}$. A straightforward argument shows that by Theorem 3.5 one finally arrives at the following system of $C_{d}^{2}$ linear equations on $d$ variables:

$$
x_{e}+x_{e^{\prime}}=\tilde{x}_{e}+\tilde{x}_{e^{\prime}} \text { for any path }\left(e, e^{\prime}\right) \text { through } V,
$$

the new variables being introduced as follows:

$$
x_{e}:=\frac{1}{\alpha_{V_{e}}} ; \quad \tilde{x}_{e}:=\frac{1}{\tilde{\alpha}_{V_{e}}},
$$

where $V_{e}$ is a vertex such that together with $V$ it constitutes the pair of endpoints of the edge $e$, incident to $V$. Clearly this system admits the only solution $x_{e}=\tilde{x}_{e}$ for any $e$ incident to the vertex $V$, provided that $d>2$.

Therefore, in the case of valences higher than 2 one not only gets $\alpha_{V}=\tilde{\alpha}_{V}$ for the vertex $V$ itself as in the case of $\operatorname{deg} V=2$, but also automatically $\alpha_{V^{\prime}}=\tilde{\alpha}_{V^{\prime}}$ for any vertex $V^{\prime}$ adjacent to $V$.

The argument presented above in fact applies to an arbitrary graph $\Gamma_{\delta}$ rather than to just a tree. One has the following 
Theorem 3.8. Assume that $A_{\vec{\alpha}}$ and $A_{\overrightarrow{\widetilde{\alpha}}}$ are two Laplacians on an arbitrary compact metric graph $\Gamma_{\delta}$ with all edges (except loops) of mixed type. Suppose that the number of vertices is greater than twd2. Assume that all edge lengths are rationally independent. Let $A_{\vec{\alpha}}$ and $A_{\overrightarrow{\widetilde{\alpha}}}$ be isospectral for $\vec{\alpha}$ and $\overrightarrow{\tilde{\alpha}}$ such that $\alpha_{i} \neq 0$ (and hence $\tilde{\alpha}_{i} \neq 0$ ) for all $i=1, \ldots, N$. Then $\alpha_{i}=\tilde{\alpha}_{i}$ for all $i=1, \ldots, N$.

Proof. The case of a tree graph has in fact been proven above. Indeed, one immediately obtains the claimed result for all internal vertices. Then one uses balancing conditions at all boundary vertices.

Consider the situation when $\Gamma_{\delta}$ has no multiple edges and no loops. Then for any vertex $V$ of the graph there exists a spanning tree $T$ such that every edge incident to $V$ belongs to it. One can then proceed analogously to the case of a tree graph, cf. the proof of Lemma 3.7.

The situation when $\Gamma_{\delta}$ is allowed to contain multiple edges is somewhat different, although can be considered in much the same way. The balancing condition (13) in particular admits the form

$$
\alpha_{V}-\sum_{V^{\prime} \text { adjacent to } V} \frac{\nu\left(V^{\prime}\right)}{\alpha_{V^{\prime}}}=\tilde{\alpha}_{V}-\sum_{V^{\prime} \text { adjacent to } V} \frac{\nu\left(V^{\prime}\right)}{\tilde{\alpha}_{V^{\prime}}}
$$

where $\nu\left(V^{\prime}\right)$ is the multiplicity of edge connection betwen $V$ and $V^{\prime}$, i.e., the number of edges having vertices $V$ and $V^{\prime}$ as their endpoints. The rest of the proof remains virtually unchanged.

Finally, one notes that the possible presence of loops does not change the argument a single bit; balancing conditions at graph vertices do not involve any loops-related information.

Remark 3. Balancing conditions (14) at the first glance look awkwardly non-linear. Nevertheless, passing over to the variables $\sigma_{j}$ defined in (11) one arrives at the following systems of balancing equations:

$$
\operatorname{deg} V \sigma_{V}+\sum_{V^{\prime} \text { adjacent to } V} \frac{\nu\left(V^{\prime}\right) \sigma_{V^{\prime}}}{\operatorname{deg} V^{\prime}}=\operatorname{deg} V \tilde{\sigma}_{V}+\sum_{V^{\prime} \text { adjacent to } V} \frac{\nu\left(V^{\prime}\right) \tilde{\sigma}_{V^{\prime}}}{\operatorname{deg} V^{\prime}}
$$

for all vertices $V$ of $\delta$ type and

$$
\frac{\operatorname{deg} V}{\sigma_{V}}+\sum_{V^{\prime} \text { adjacent to } V} \frac{\nu\left(V^{\prime}\right)}{\operatorname{deg} V^{\prime} \sigma_{V^{\prime}}}=\frac{\operatorname{deg} V}{\tilde{\sigma}_{V}}+\sum_{V^{\prime} \text { adjacent to } V} \frac{\nu\left(V^{\prime}\right)}{\operatorname{deg} V^{\prime} \tilde{\sigma}_{V^{\prime}}}
$$

for all vertices $V$ of $\delta^{\prime}$ type, which are linear with respect to $\left\{\sigma_{i}\right\}$ and $\left\{1 / \sigma_{i}\right\}$, respectively.

\footnotetext{
${ }^{2}$ Clearly the degenerate case of $N=2$ leads to trivial isospectrality since one can always swap both coupling constants which leads to the same graph Laplacian.
} 
We now sum up all the information obtained so far pertaining to the question of whether or not isospectrality yields (9) in the form of the following

Corollary 3.9. Assume that $A_{\vec{\alpha}}$ and $A_{\vec{\alpha}}$ are two isospectral Laplacians on the graph $\Gamma_{\delta}$. Then if $\Gamma_{\delta}$ is either

(i) a graph with all vertices of $\delta$ type or a graph with all vertices of $\delta^{\prime}$ type,

or (ii) a non-tree graph with vertices of different types,

or (iii) a tree with all edges of mixed type and the additional condition $\alpha_{i} \neq 0$ (and hence $\tilde{\alpha}_{i} \neq 0$ ) for all $i=1, \ldots, N$,

condition (9) holds.

Tightness of this result is demonstrated by the following

Example 1. Let $\Gamma_{\delta}=A_{3}$, i.e., the chain graph of three vertices, with rationally independent edge lengths. Let the types of its vertices be either $\delta-\delta^{\prime}-\delta$ or $\delta^{\prime}-\delta-\delta^{\prime}$. In both cases there exist non-trivial isospectral configurations of coupling constants, leading to $\Phi(\vec{\alpha})=-\Phi(\overrightarrow{\tilde{\alpha}})$.

Proof. A direct application of Theorem 3.5 gives the following description of all isospectral configurations for the case $\delta-\delta^{\prime}-\delta$ :

$$
\vec{\alpha}=\left(a, \frac{2}{a}, 0\right) ; \quad \overrightarrow{\tilde{\alpha}}=\left(0,-\frac{2}{a},-a\right) \text { for all } a \in \mathbb{R} .
$$

The second case is analogous.

\section{Graph Reductions}

The aim of the present Section is to demonstrate how one can reduce the problem of isospectrality for graph Laplacians defined on $\Gamma_{\delta}$ to the consideration of "smaller", or trimmed graphs. In particular, we will show how the general situation of mixed $\delta$ and $\delta^{\prime}$ type vertices can be reduced to the case considered towards the end of the Section 3, i.e., to the case when all graph edges are of mixed type.

The reductions we have in mind are actually threefold: (i) trimming away a boundary vertex together with the edge it belongs to; (ii) trimming away any internal edge, be it simple or multiple; (iii) trimming away a loop attached to any graph vertex.

In all these three cases the analysis is in fact very similar. It is based on explicit comparison of residues of $\operatorname{det}(M(\lambda)-B)$ and $\operatorname{det}(M(\lambda)-\tilde{B})$ using results of Section 3 and in particular Theorem 3.5.

We start with the following setup. Let $\Gamma_{\delta}$ be an arbitrary compact metric marked graph. Assume that two Laplacians $A_{\vec{\alpha}}$ and $A_{\overrightarrow{\tilde{\alpha}}}$ are isospectral on this graph. Further assume that the graph contains an 
edge $e$ with both endpoints of $\delta$ type. Consider the graph $\Gamma_{\delta}^{(e)}=\Gamma_{\delta}-e$, i.e., the graph $\Gamma_{\delta}$ with the edge $e$ removed. This means, that the vertices $V_{j}$ and $V_{k}$ connected in the original graph by the edge $e$ have been "glued" together to form the vertex $V_{j, k}^{(e)}$ (of the same type $\delta$ ), whereas the rest of the graph remains unchanged. We do allow the situation of multiple edges, when $V_{j}$ and $V_{k}$ are connected in $\Gamma_{\delta}$ by more than one edge. In this situation all these edges, but the edge $e$, become nothing but loops of their respective lengths, attached to the vertex $V_{j, k}^{(e)}$. We are going to argue that under the assumption of isospectrality of $A_{\vec{\alpha}}$ and $A_{\vec{\alpha}}$ one is guaranteed isospectrality of $A_{\overrightarrow{\alpha_{e}}}^{(e)}$ and $A_{\vec{\alpha}_{e}}^{(e)}$, where these two operators are graph Laplacians defined on $\Gamma_{\delta}^{(e)}$ with vectors of coupling constants $\vec{\alpha}_{e}$ and $\overrightarrow{\tilde{\alpha}}_{e}$, respectively. These vectors in turn are from $\mathbb{R}^{N-1}$ and are constructed based on $\vec{\alpha}$ and $\overrightarrow{\tilde{\alpha}}$ by the following rule: a coupling constant at any unaffected vertex remains the same, whereas the coupling constants at $V_{j, k}^{(e)}$ are $\alpha_{j}+\alpha_{k}$ and $\tilde{\alpha}_{j}+\tilde{\alpha}_{k}$, respectively.

The argument goes as follows. Having assumed isospectrality, one can use the result of Lemma 3.2. Having divided both sides of (4) by $\Pi(\lambda)$ away from poles of determinants, one can now compute residues of both sides at the sequence of first order (by the condition of rational independence of edge lengths) poles $\mu_{e}(m), m=1,2, \ldots$, associated with the length $l$ of the edge $e$. These poles are obviously located at zeroes of $\sin l \mu$. The named residues then must coincide as well.

For any of the two determinants, one adds the $k$-th row of the matrix $M(\lambda)-B$ multiplied by $\cos l \mu$ to the $j$-th one. By Proposition 2.1, this cancels out singularities of all matrix elements of the $j$-th row at the sequence $\mu_{e}(m)$. Then the residues are calculated by passing to the limit as $\mu \rightarrow \mu_{e}(m)$ in $\left(\mu+\mu_{e}(m)\right)\left(\mu-\mu_{e}(m)\right) \operatorname{det}(M(\lambda)-B)$, where the factor $\left(\mu-\mu_{e}(m)\right)$ is introduced into the $k$-th row of the matrix since by inspection all other rows are now regular at the sequence of points $\mu_{e}(m)$. Thereafter, we add the $k$-th column to the $j$-th one and, having noted that the $k$-th row now only contains a single nonzero element, reduce the determinant to the one of a matrix of lower dimension.

The procedure outlined above yields:

$$
\Phi(\vec{\alpha}) \operatorname{det}\left(M^{(e)}\left(\mu_{e}^{2}(m)\right)-B_{e}\right)=\Phi(\overrightarrow{\tilde{\alpha}}) \operatorname{det}\left(M^{(e)}\left(\mu_{e}^{2}(m)\right)-\tilde{B}_{e}\right) \quad \forall m,
$$

where $M^{(e)}(\lambda)$ is the Weyl-Titchmarsh $M$-matrix of the graph $\Gamma_{\delta}^{(e)}$ defined above, $B_{e}$ and $\tilde{B}_{e}$ are two diagonal matrices of coupling constants $\vec{\alpha}_{e}$ and $\overrightarrow{\alpha_{e}}$, respectively. 
Note that $\Phi(\vec{\alpha})=\Phi_{e}\left(\vec{\alpha}_{e}\right)$ and $\Phi(\overrightarrow{\tilde{\alpha}})=\Phi_{e}\left(\overrightarrow{\tilde{\alpha}}_{e}\right)$ since the set of $\delta^{\prime}$ vertices and their respective coupling constants are the same for $\Gamma_{\delta}$ and $\Gamma_{\delta}^{(e)}$ by construction. Here the function $\Phi_{e}$ is defined by the same expression (5) as the function $\Phi$, but for the modified graph $\Gamma_{\delta}^{(e)}$.

By Lemma 3.2 again, it remains to be seen that the equality (15) holds everywhere in $\mathbb{C}$ with the exception of the countable set of poles rather than just at the sequence of points $\mu_{e}(m)$. In order to ascertain this, we first multiply both sides of (15) by $\Pi_{e}\left(\mu_{e}^{2}(m)\right)$, where the function $\Pi_{e}$ is defined by the same expression (3) as the function $\Pi$, but for the graph $\Gamma_{\delta}^{(e)}$. By Theorem 3.4, one immediately gets the following expansion in powers of $m$ :

$$
\begin{aligned}
\Phi_{e}\left(\overrightarrow{\alpha_{e}}\right) \Pi_{e}\left(\mu_{e}^{2}(m)\right) \operatorname{det}\left(M^{(e)}\right. & \left.\left(\mu_{e}^{2}(m)\right)-B_{e}\right) \\
& =\sum_{j} m^{j} \Psi_{j}\left(\overrightarrow{\alpha_{e}} ; l_{1} \mu_{e}(m), \ldots, l_{n-1} \mu_{e}(m)\right)
\end{aligned}
$$

with functions $\Psi_{j}$ which are linear combinations (with coefficients depending on $\overrightarrow{\alpha_{e}}$ ) of various products of sines and cosines of different arguments, belonging to the set $\left\{l_{1} \mu_{e}(m), \ldots, l_{n-1} \mu_{e}(m)\right\}$. Here $l_{1}, \ldots, l_{n-1}$ are (with a slight abuse of notation) the collection of edge lengths pertaining to the graph $\Gamma_{\delta}^{(e)}$ and are thus rationally independent.

Let $j_{0}$ be the topmost power of $m$ in the above representation and consider the function $\Psi_{j_{0}}\left(\overrightarrow{\alpha_{e}} ; l_{1} \mu_{e}(m), \ldots, l_{n-1} \mu_{e}(m)\right)$. On the one hand, an application of (15) immediately yields

$$
\begin{aligned}
\lim _{m \rightarrow \infty} \Psi_{j_{0}}\left(\overrightarrow{\alpha_{e}} ; l_{1} \mu_{e}(m), \ldots,\right. & \left.l_{n-1} \mu_{e}(m)\right) \\
& =\lim _{m \rightarrow \infty} \Psi_{j_{0}}\left(\overrightarrow{\tilde{\alpha}_{e}} ; l_{1} \mu_{e}(m), \ldots, l_{n-1} \mu_{e}(m)\right)
\end{aligned}
$$

On the other hand, consider an analytic [31] function $\Theta_{j_{0}}$ of $n-1$ complex variables parametrically depending on $\overrightarrow{\alpha_{e}}$ which is defined in the following way:

$$
\Theta_{j_{0}}\left(z_{1}, \ldots, z_{n-1} ; \overrightarrow{\alpha_{e}}\right):=\Psi_{j_{0}}\left(\overrightarrow{\alpha_{e}} ; z_{1}, \ldots, z_{n-1}\right) .
$$

Note that this function is in fact $2 \pi$-periodic with respect to any of its variables restrained to the real line.

We will now use a result of [7] which says that for $n-1$ rationally independent real values $\hat{l}_{1}, \ldots, \hat{l}_{n-1}$ (here we have put $\hat{l}_{j}:=l_{j} / 2 l$, where $l$ is the length of the edge $e)$ the set of points $\left(\left\{m \hat{l}_{1}\right\}, \ldots,\left\{m \hat{l}_{n-1}\right\}\right)$, where $\{x\}$ denotes the fractional part of $x$ and $m$ is a natural number, is dense in the unit cube $\mathbb{K}_{1} \subset \mathbb{R}^{n-1}$. 
Since $\mu_{e}(m)=\pi m / l$, one then has using Hartogs' theorem the following identities for any real $z_{1}, \ldots, z_{n}$ in $2 \pi \mathbb{K}_{1} \subset \mathbb{R}^{n-1}$ and a subsequence $\left\{m_{k}\right\}_{k=1}^{\infty}$ :

$$
\begin{gathered}
\Theta_{j_{0}}\left(z_{1}, \ldots, z_{n-1} ; \overrightarrow{\alpha_{e}}\right)=\lim _{k \rightarrow \infty} \Theta_{j_{0}}\left(2 \pi\left\{\hat{l}_{1} m_{k}\right\}, \ldots,\left\{\hat{l}_{n-1} m_{k}\right\} ; \overrightarrow{\alpha_{e}}\right)= \\
\lim _{k \rightarrow \infty} \Theta_{j_{0}}\left(l_{1} \mu_{e}\left(m_{k}\right), \ldots, l_{n-1} \mu_{e}\left(m_{k}\right) ; \overrightarrow{\alpha_{e}}\right)= \\
\lim _{k \rightarrow \infty} \Psi_{j_{0}}\left(\overrightarrow{\alpha_{e}} ; l_{1} \mu_{e}\left(m_{k}\right), \ldots, l_{n-1} \mu_{e}\left(m_{k}\right)\right)= \\
\lim _{k \rightarrow \infty} \Psi_{j_{0}}\left(\overrightarrow{\overrightarrow{\alpha_{e}}} ; l_{1} \mu_{e}\left(m_{k}\right), \ldots, l_{n-1} \mu_{e}\left(m_{k}\right)\right)=\Theta_{j_{0}}\left(z_{1}, \ldots, z_{n-1} ; \overrightarrow{\tilde{\alpha}_{e}}\right),
\end{gathered}
$$

where the last equality follows by reversing the first three.

Thus,

$$
\Theta_{j_{0}}\left(z_{1}, \ldots, z_{n-1} ; \overrightarrow{\alpha_{e}}\right)=\Theta_{j_{0}}\left(z_{1}, \ldots, z_{n-1} ; \overrightarrow{\alpha_{e}}\right)
$$

everywhere in $2 \pi \mathbb{K}_{1}$, from where by the corresponding uniqueness theorem [31] it follows immediately, that the last equality holds everywhere in $\mathbb{C}^{n-1}$. In particular, it holds on the ray $z_{1}=l_{1} \mu, z_{2}=l_{2} z_{1} / l_{1}, \ldots$, $z_{n-1}=l_{n-1} z_{1} / l_{1}$, which finally yields

$$
\Psi_{j_{0}}\left(\overrightarrow{\alpha_{e}} ; l_{1} \mu, \ldots, l_{n-1} \mu\right)=\Psi_{j_{0}}\left(\overrightarrow{\tilde{\alpha}_{e}} ; l_{1} \mu, \ldots, l_{n-1} \mu\right) \quad \forall \mu \in \mathbb{C} .
$$

Now repeating the argument first for $\Psi_{j_{1}}$, where $j_{1}$ is the second highest power of $\mu$, and then for all the other consecutive powers, one obtains that

$$
\Psi_{j}\left(\overrightarrow{\alpha_{e}} ; l_{1} \mu, \ldots, l_{n-1} \mu\right)=\Psi_{j}\left(\overrightarrow{\tilde{\alpha}_{e}} ; l_{1} \mu, \ldots, l_{n-1} \mu\right) \quad \forall \mu \in \mathbb{C} \text { and } \forall j .
$$

Ultimately, writing down the corresponding expansion in powers of $\mu$ for $\Phi_{e}\left(\overrightarrow{\alpha_{e}}\right) \Pi_{e}\left(\mu^{2}\right) \operatorname{det}\left(M^{(e)}\left(\mu^{2}\right)-B_{e}\right)$ and comparing it with the one for $\Phi_{e}\left(\overrightarrow{\tilde{\alpha}}_{e}\right) \Pi_{e}\left(\mu^{2}\right) \operatorname{det}\left(M^{(e)}\left(\mu^{2}\right)-\tilde{B}_{e}\right)$, one ascertains the identity sought:

$$
\Phi_{e}\left(\vec{\alpha}_{e}\right) \Pi_{e}(\lambda) \operatorname{det}\left(M^{(e)}(\lambda)-B_{e}\right)=\Phi_{e}\left(\overrightarrow{\tilde{\alpha}}_{e}\right) \Pi_{e}(\lambda) \operatorname{det}\left(M^{(e)}(\lambda)-\tilde{B}_{e}\right),
$$

for all $\lambda$, which completes the proof of the claim.

The corresponding result in the case of $e$ being an edge with both endpoints of $\delta^{\prime}$ type follows from a virtually unchanged argument. The only bit that probably deserves a comment is the following one. The identities $\Phi(\vec{\alpha})=\Phi_{e}\left(\vec{\alpha}_{e}\right)$ and $\Phi(\overrightarrow{\tilde{\alpha}})=\Phi_{e}\left(\overrightarrow{\tilde{\alpha}_{e}}\right)$ in this setup follow from (12). This identity immediately implies that the statement of Lemma 3.2 holds if one replaces the factor $\Phi$ on both sides by the function $\hat{\Phi}$, where

$$
\hat{\Phi}(\vec{\alpha}):=\prod_{V_{j} \text { of } \delta \text { type: } \alpha_{j} \neq 0} \frac{\operatorname{deg} V_{j}}{\alpha_{j}} .
$$


Then the identities in question follow immediately since now the set of $\delta$ type vertices and their respective coupling constants are the same for $\Gamma_{\delta}$ and $\Gamma_{\delta}^{(e)}$ by construction.

We now shift our attention to the case when $e$ is a loop attached to the vertex $V$. The analysis of this situation is if anything simpler than the one presented above. In this setup, one has to consider the modified graph $\Gamma_{\delta}^{(e)}=\Gamma_{\delta}-V$, that is, the graph $\Gamma_{\delta}$ with the vertex $V$ removed together with all graph edges incident to it (including the loop $e$, of course). The modified vector of coupling constants $\overrightarrow{\alpha_{e}}$ is nothing but the vector $\vec{\alpha}$ with the element pertaining to the vertex $V$ dropped (the same modification yields $\overrightarrow{\alpha_{e}}$, of course). In order to ensure that $\Gamma_{\delta}^{(e)}$ is connected, we additionally require that the vertex $V$ in question either belongs to the graph boundary or to a cycle of $\Gamma_{\delta}$.

Assume w.l.o.g. that $V$ is of $\delta$ type. The residue calculation then trivially yields:

$\Phi_{e}\left(\vec{\alpha}_{e}\right) \operatorname{det}\left(M^{(V)}\left(\mu_{e}^{2}(m)\right)-B_{e}\right)=\Phi_{e}\left(\overrightarrow{\tilde{\alpha}}_{e}\right) \operatorname{det}\left(M^{(V)}\left(\mu_{e}^{2}(m)\right)-\tilde{B}_{e}\right) \quad \forall m$,

where as above $B_{e}$ and $\tilde{B}_{e}$ are two diagonal matrices of coupling constants $\vec{\alpha}_{e}$ and $\overrightarrow{\tilde{\alpha}}_{e}$, respectively. The matrix $M^{(V)}$ however is not a Weyl-Titchmarsh matrix of any graph since it is actually equal to $M$ in which the row and the column pertaining to the vertex $V$ have been dropped.

It is nevertheless possible by the same line of argumentation as above to ascertain the identity

$$
\Phi_{e}\left(\vec{\alpha}_{e}\right) \Pi_{e}(\lambda) \operatorname{det}\left(M^{(V)}(\lambda)-B_{e}\right)=\Phi_{e}\left(\overrightarrow{\tilde{\alpha}}_{e}\right) \Pi_{e}(\lambda) \operatorname{det}\left(M^{(V)}(\lambda)-\tilde{B}_{e}\right)
$$

for all $\lambda \in \mathbb{C}$, where $\Pi_{e}$ has the same meaning as in the analysis presented above. Then one is able to expand the determinant as

$$
\operatorname{det}\left(M^{(V)}(\lambda)-B_{e}\right)=\operatorname{det}\left(M^{(e)}(\lambda)-B_{e}\right)+\Omega(\lambda),
$$

where $M^{(e)}(\lambda)$ is the Weyl-Titchmarsh matrix of the graph $\Gamma_{\delta}^{(e)}$, whence by the linear independence argument (using the fact that $\Omega(\lambda)$ is sure to contain trigonometric functions with arguments not to be found in the expression for $M^{(e)}$ ) one ultimately has the identity sought

$$
\Phi_{e}\left(\vec{\alpha}_{e}\right) \Pi_{e}(\lambda) \operatorname{det}\left(M^{(e)}(\lambda)-B_{e}\right)=\Phi_{e}\left(\overrightarrow{\tilde{\alpha}}_{e}\right) \Pi_{e}(\lambda) \operatorname{det}\left(M^{(e)}(\lambda)-\tilde{B}_{e}\right)
$$

$\forall \lambda \in \mathbb{C}$. In order to give precise formulation of results obtained so far, we start with the following

Definition 3. Either of the following graph operations on $\Gamma_{\delta}$ will be called an admissible trimming of the graph: 
(i) a removal of an edge connecting two different vertices $V$ and $V^{\prime}$ of the same type, $\Gamma_{\delta}^{(e)}=\Gamma_{\delta}-e$;

(ii) a removal of a vertex $V$ to which a loop $e$ is attached provided that this vertex either belongs to the graph boundary or to one of the graph cycles, $\Gamma_{\delta}^{(e)}=\Gamma_{\delta}-V$.

Then the following Theorem holds.

Theorem 4.1. Assume that $A_{\vec{\alpha}}$ and $A_{\overrightarrow{\widetilde{\alpha}}}$ are two Laplacians on the graph $\Gamma_{\delta}$. Assume that all the edge lengths are rationally independent. Finally, let $A_{\vec{\alpha}}$ and $A_{\vec{\alpha}}$ be isospectral. Then for any admissible trimming $\Gamma_{\delta}^{(e)}$ of the graph $\Gamma_{\delta}$, the Laplacians $A_{\overrightarrow{\alpha_{e}}}$ and $\overrightarrow{A_{\overrightarrow{\alpha_{e}}}}$ on the trimmed graph are isospectral.

Here the vectors of coupling constants $\overrightarrow{\alpha_{e}}$ and $\overrightarrow{\tilde{\alpha}_{e}}$ are: in the case of an admissible trimming (i) equal to the coupling constants of $A_{\vec{\alpha}}$ and $A_{\overrightarrow{\widetilde{\alpha}}}$, respectively, for all vertices except $V$ and $V^{\prime}$ with the remaining ones being equal to $\alpha_{V}+\alpha_{V^{\prime}}$ and $\tilde{\alpha}_{V}+\tilde{\alpha}_{V^{\prime}}$, respectively; in the case of an admissible trimming (ii) equal to the coupling constants of $A_{\vec{\alpha}}$ and $A_{\overrightarrow{\widetilde{\alpha}}}$, respectively, for all vertices except $V$.

Remark 4. 1. Note that a procedure of trimming away graph boundary vertices was suggested in a similar context in [26]. The latter Theorem provides a much more general recipe of this procedure, allowing in particular to deal with graph cycles.

2. Theorem 4.1 in the case of mixed $\delta$ and $\delta^{\prime}$ type vertices clearly allows to reduce the analysis of isospectral Laplacians to the setup studied towards the end of Section 3, i.e., when all graph edges are assumed to be of mixed type.

3. Trimming away an edge when one of its endpoints $V$ has $\alpha_{V}=$ $\tilde{\alpha}_{V}=0$ yields more information, see Theorem 4.7 below.

The following immediate Corollary of Theorem4.1demonstrates how one could use this result in order to prove uniqueness (i.e., the absence of isospectral configurations of coupling constants).

Corollary 4.2. Assume that there exist either two different admissible trimmings of the same type or a sequence of two consecutive admissible trimmings of type (i) for the graph $\Gamma_{\delta}$. Let each of these two trimmings result in a graph $\Gamma_{\delta}^{(e)}$ for which the absence of isospectral configuration of coupling constants is known. Then there are no isospectral configurations for the graph $\Gamma_{\delta}$.

Results obtained in [12, Section 5] now yield 
Corollary 4.3. If $\Gamma_{\delta}$ is a tree graph with all vertices of the same type (i.e., either of type $\delta$ or of type $\delta^{\prime}$ ), the spectrum of a graph Laplacian defined on $\Gamma_{\delta}$ uniquely determines all coupling constants.

A further corollary of Theorem 4.1 shows that in the process of graph trimming any cycles, multiple edges and loops (note that graph cycles reduce to multiple edges, which in turn reduce to loops under consecutive trimmings) are in fact helpful if one wishes to exclude the potentially problematic situation of Example 1, whereby some coupling constants are allowed to zero out.

Corollary 4.4. Assume that $A_{\vec{\alpha}}$ and $A_{\overrightarrow{\widetilde{\alpha}}}$ are two Laplacians on the graph $\Gamma_{\delta}$. Assume that all the edge lengths are rationally independent. Let the graph contain a vertex $V$ such that: (i) $V$ has a loop attached to it and (ii) $\alpha_{V}=0, \tilde{\alpha}_{V} \neq 0$. Then $A_{\vec{\alpha}}$ and $A_{\overrightarrow{\widetilde{\alpha}}}$ cannot be isospectral.

Proof. Assume the opposite. Then Theorem 4.1 provides isospectrality for the corresponding graph Laplacians on $\Gamma_{\delta}-V$. One immediately arrives at a contradiction, since isospectrality requires that the total number of zero coupling constants has to be the same.

The following Example demonstrates how Theorem 4.1 can be used to single out isospectral configurations provided that these in fact exist.

Example 2. Consider the graph $\Gamma_{\delta}$ which is a pure cycle of 4 vertices with rationally independent edge lengths. Let all vertices be of type $\delta$.

On the one hand, it is known [12 that in this situation there are isospectral configurations of coupling constants, namely, for an arbitrary non-zero $a \in \mathbb{R}, \vec{\alpha}=(a,-a, a,-a), \overrightarrow{\tilde{\alpha}}=(-a, a,-a, a)$, and for these configurations only the corresponding graph Laplacians are isospectral.

On the other hand, Theorem 4.1 and Corollary 4.2 immediately imply, that either there are no isospectral configurations by the result [12] pertaining to the case of odd cycles, or $\alpha_{1}+\alpha_{2}=0=\tilde{\alpha}_{1}+\tilde{\alpha}_{2}$ (in which case the graph $\Gamma_{\delta}^{(e)}$ reduces by the procedure of cleaning, see [26], to a cycle of two vertices for which one might easily swap the coupling constants with no effect on the spectrum as explained above). The remaining three admissible trimmings of $\Gamma_{\delta}$ leave $\alpha_{2}+\alpha_{3}=0=\tilde{\alpha}_{2}+\tilde{\alpha}_{3}$, $\alpha_{3}+\alpha_{4}=0=\tilde{\alpha}_{3}+\tilde{\alpha}_{4}$ and finally $\alpha_{1}+\alpha_{4}=0=\tilde{\alpha}_{1}+\tilde{\alpha}_{4}$ for possible isospectral configurations. These four relations clearly yield precisely the result of [12].

The remaining part of the paper is devoted to the analysis of isospectrality for graph Laplacians in the situation when all graph edges of $\Gamma_{\delta}$ are of mixed type, i.e., when for each edge one of the endpoints is 
of type $\delta$ whereas the other one is of $\delta^{\prime}$ type. The general situation can be reduced to the one outlined by consecutive applications of Theorem 4.1. As no further trimming is possible once we have arrived at a graph of this class, one has no choice but to resort to Theorem 3.5. Nevertheless, there is room still for simplifications based on residue calculus.

We are going to argue that in this case there are no isospectral graph Laplacians unless the underlying graph is essentially the graph of Example 1, i.e., the chain graph of exactly 3 vertices.

We use the following notation throughout for the sake of brevity. Having fixed two configurations of coupling constants, $\vec{\alpha}$ and $\overrightarrow{\tilde{\alpha}}$, which by assumption lead to isospectral graph Laplacians, we will say that a graph vertex $V$ is of $0 \overline{0}$ class iff $\alpha_{V}=0$ and $\tilde{\alpha}_{V} \neq 0$; is of $\overline{0} 0$ class iff $\alpha_{V} \neq 0$ and $\tilde{\alpha}_{V}=0$; is of 00 class iff $\alpha_{V}=0$ and $\tilde{\alpha}_{V}=0$ and finally is of $\overline{0} \overline{0}$ class iff $\alpha_{V} \neq 0$ and $\tilde{\alpha}_{V} \neq 0$. In these terms, Theorem 3.8 reads: if all graph vertices are of $\overline{0} \overline{0}$ class, one has $\vec{\alpha}=\overrightarrow{\tilde{\alpha}}$.

As demonstrated by Example 1, the situation when coupling constants at graph vertices are allowed to zero out can in fact lead to complications, including the possibility that the condition (9) is not satisfied. As shown by the named example, this in fact is due to the presence of vertices of $0 \overline{0}$ and $\overline{0} 0$ classes.

We start however with consideration of 00 class of vertices. The residue-based analysis used above will allow us to reduce the general situation to the one when one is sure to have no vertices of this class. The problem we face is that residue calculation in fact removes an edge rather than a vertex as such; on the other hand, in the situation considered endpoints of any given edge are of different types. It is not feasible to expect that a removal of this edge will as above leave us with an $M$-function of any $\Gamma_{\delta}$. In order to give a description of what is to happen here, introduce the following notion.

Definition 4. (i) Removal of a mixed edge giving rise to a vertex of $\delta^{\prime} \rightarrow \delta$ type. The graph $\Gamma_{\delta}$ is subject to the following modification resulting in the quasigraph $\hat{\Gamma}_{\delta}^{(e)}$. An edge $e$ connecting vertices $V$ and $V^{\prime}$ of types $\delta$ and $\delta^{\prime}$, respectively, is removed. The vertices $V$ and $V^{\prime}$ are glued together to form a quasivertex $\hat{V}$ of $\delta^{\prime} \rightarrow \delta$ type. Any other edge $e^{\prime}$ of length $l^{\prime}$ connecting $V$ and $V^{\prime}$ in $\Gamma_{\delta}$ gives rise to a quasiloop of the same length $l^{\prime}$ attached to $\hat{V}$. Any loop $\mathcal{O}$ attached to $V^{\prime}$ becomes a quasiloop of the same length attached to $\hat{V}$. Any edge $e^{\prime}$ incident to $V^{\prime}$ and $V^{\prime \prime} \neq V$ in $\Gamma_{\delta}$ becomes a quasiedge of the same length connecting $\hat{V}$ and $V^{\prime \prime}$. The rest of $\Gamma_{\delta}$ remains unchanged. 
(ii) Removal of a mixed edge giving rise to a vertex of $\delta \rightarrow \delta^{\prime}$ type. The graph $\Gamma_{\delta}$ is subject to the following modification resulting in the quasigraph $\hat{\Gamma}_{\delta}^{(e)}$. An edge $e$ connecting vertices $V$ and $V^{\prime}$ of types $\delta$ and $\delta^{\prime}$, respectively, is removed. The vertices $V$ and $V^{\prime}$ are glued together to form a quasivertex $\hat{V}$ of $\delta \rightarrow \delta^{\prime}$ type. Any other edge $e^{\prime}$ of length $l^{\prime}$ connecting $V$ and $V^{\prime}$ in $\Gamma_{\delta}$ gives rise to a quasiloop of the same length $l^{\prime}$ attached to $\hat{V}$. Any loop $\mathcal{O}$ attached to $V$ becomes a quasiloop of the same length attached to $\hat{V}$. Any edge $e^{\prime}$ incident to $V$ and $V^{\prime \prime} \neq V^{\prime}$ in $\Gamma_{\delta}$ becomes a quasiedge of the same length connecting $\hat{V}$ and $V^{\prime \prime}$. The rest of $\Gamma_{\delta}$ remains unchanged.

We point out that we do not allow the procedure outlined above to be applied to more than a single edge $e$ of a mixed type. In other words, we do not apply Definition 4 consecutively.

Note that the difference between the procedures (i) and (ii) defined above is essentially the following: one either eliminates the vertex $V^{\prime}$ by moving it to the vertex $V$ along the edge $e$, which forces all other edges incident to $V^{\prime}$ in $\Gamma_{\delta}$ to acquire the prefix "quasi", or eliminates $V$ by moving it to $V^{\prime}$ with an analogous effect. This makes the notation $\delta^{\prime} \rightarrow \delta$ and $\delta \rightarrow \delta^{\prime}$ chosen by us self-explanatory. It is obvious that Definition 4 gives rise to two different types of quasiedges, depending on the type of quasivertex these are incident to. Since $\Gamma_{\delta}$ by assumption only contains edges of mixed type, a quasiedge $e$ incident to a quasivertex of $\delta^{\prime} \rightarrow \delta$ type must be also incident to a vertex of $\delta$ type; a quasiedge $e$ incident to a quasivertex of $\delta \rightarrow \delta^{\prime}$ type must be also incident to a vertex of $\delta^{\prime}$ type. We will further need the following

Definition 5. An $M$-matrix $\hat{M}^{(e)}(\mu)$ of a quasigraph $\hat{\Gamma}_{\delta}^{(e)}$ is defined as follows (cf. (11) and (2)):

(i) If $V_{k}$ is a vertex of $\delta$ type or a quasivertex of $\delta^{\prime} \rightarrow \delta$ type, $\hat{m}_{j k}(\mu)=$

$$
\begin{cases}\mu\left(\sum_{e_{t} \in \hat{E}_{k}^{\prime}} \tan \mu l_{t}+2 \sum_{e_{t} \in L_{k}} \tan \frac{\mu l_{t}}{2}\right. & \\ \left.-2 \sum_{e_{t} \in \hat{L}_{k}} \cot \left(\frac{\pi}{4}+\frac{\mu l_{t}}{2}\right)-2 \sum_{e_{t} \in \hat{L}_{k}^{\prime}} \cot \frac{\mu l_{t}}{2}\right), & j=k, \\ -\mu \sum_{e_{t} \in \hat{C}_{k j}} \frac{1}{\cos \mu l_{t}}-\sum_{e_{t} \in C_{k j}^{\prime}} \frac{1}{\cos \mu l_{t}}, & j \neq k, V_{j} \text { is } \\ 0, & \text { adjacent to } V_{k}, \\ & j \neq k, V_{j} \text { is } \\ & \text { not adjacent to } V_{k} ;\end{cases}
$$


(ii) If $V_{k}$ is a vertex of $\delta^{\prime}$ type or a quasivertex of $\delta \rightarrow \delta^{\prime}$ type, $\hat{m}_{j k}(\mu)=$

$$
\begin{cases}\frac{1}{\mu}\left(\sum_{e_{t} \in \hat{E}_{k}^{\prime}} \tan \mu l_{t}-2 \sum_{e_{t} \in L_{k}} \cot \frac{\mu l_{t}}{2}\right. & \\ \left.-2 \sum_{e_{t} \in \hat{L}_{k}} \cot \left(\frac{\pi}{4}+\frac{\mu l_{t}}{2}\right)+2 \sum_{e_{t} \in \hat{L}_{k}^{\prime}} \tan \frac{\mu l_{t}}{2}\right), & j=k, \\ -\sum_{e_{t} \in C_{k j}^{\prime}} \frac{1}{\cos \mu l_{t}}-\frac{1}{\mu} \sum_{e_{t} \in \hat{C}_{k j}} \frac{1}{\cos \mu l_{t}}, & j \neq k, V_{j} \text { is } \\ 0, & \text { adjacent to } V_{k}, \\ & j \neq k, V_{j} \text { is } \\ & \text { not adjacent to } V_{k} .\end{cases}
$$

Here $L_{k}$ is the set of loops at the (quasi)vertex $V_{k}, \hat{L}_{k}$ is the set of quasiloops at $V_{k}$ coming from elimination of multiple edges, $\hat{L}_{k}^{\prime}$ is the set of quasiloops at $V_{k}$ coming from loops at the eliminated vertex, $\hat{E}_{k}^{\prime}$ is the set of graph (quasi)edges incident to the (quasi)vertex $V_{k}, \hat{C}_{k j}$ is the set of quasiedges connecting (quasi)vertex $V_{k}$ with $V_{j}$, and finally, $C_{k j}^{\prime}$ is the set of graph edges connecting (quasi)vertex $V_{k}$ with $V_{j}$. Surely, for any pair of adjacent $V_{k}, V_{j} \in \hat{\Gamma}_{\delta}^{(e)}$ one either has $\hat{C}_{k j}=\emptyset$ or $C_{k j}^{\prime}=\emptyset$ since all connections between them are either edges or quasiedges by construction.

We remark that for all $k$ and $j$ (including $k=j$ ) such that $V_{k}$ and $V_{j}$ are "normal" graph vertices this definition is nothing but (11) and (2), where we have used the assumption that all graph edges are of mixed type. As for the situation when either $V_{k}$ or $V_{j}$ is in fact a quasivertex, one notes that quasiedges have (in terms of powers of $\mu$ ) a similar effect to regular edges and loops of $\delta-\delta$ and $\delta^{\prime}-\delta^{\prime}$ types.

From the analytic point of view however the object just defined is drastically different to the Weyl-Titchmarsh $M$-matrix of the graph $\Gamma_{\delta}$ since it is not meromorphic in the $\lambda$ plane. It is nonetheless meromorphic on the Riemann surface of $\sqrt{\lambda}$ (or, in other words, in $\mu$ plane), which is sufficient for our needs.

One has the following

Lemma 4.5. Assume that $\Gamma_{\delta}$ is a graph of more than two vertices with all edges (except loops) of "mixed" type. Assume that $A_{\vec{\alpha}}$ and $A_{\overrightarrow{\tilde{\alpha}}}$ are two isospectral graph Laplacians on $\Gamma_{\delta}$. Let $V$ and $V^{\prime}$ be $\delta$ and $\delta^{\prime}$ endpoints, respectively, of an edge e. Finally let $\hat{\Gamma}_{\delta}^{(e)}$ be constructed by either of two ways described in Definition 4. Then the identity

$$
\Phi(\vec{\alpha}) \operatorname{det}\left(\hat{M}^{(e)}(\mu)-\hat{B}_{e}\right)=\Phi(\overrightarrow{\tilde{\alpha}}) \operatorname{det}\left(\hat{M}^{(e)}(\mu)-\hat{\tilde{B}}_{e}\right)
$$


holds for all $\mu \in \mathbb{C}$ except for the countable set of poles of $\hat{M}^{(e)}(\mu)$. Here $\hat{M}^{(e)}$ is the $M$-matrix of the quasigraph $\hat{\Gamma}_{\delta}^{(e)}$, whereas $\hat{B}_{e}$ and $\hat{\tilde{B}}_{e}$ are diagonal $(N-1) \times(N-1)$ matrices of coupling constants $\vec{\beta}$ and $\overrightarrow{\tilde{\beta}}$, respectively, associated with all (quasi)vertices of $\hat{\Gamma}_{\delta}$ and defined as follows:

(i) (if one removes $e$ to form a quasivertex $\hat{V}$ of $\delta^{\prime} \rightarrow \delta$ type) $\beta_{V}=$ $\alpha_{V}, \tilde{\beta_{V}}=\tilde{\alpha_{V}}$ for any vertex $V$ of $\hat{\Gamma}_{\delta}^{(e)}$ and $\beta_{\hat{V}}=\alpha_{V}+\mu^{2} \alpha_{V^{\prime}}, \widetilde{\beta_{\hat{V}}}=$ $\tilde{\alpha}_{V}+\mu^{2} \tilde{\alpha}_{V^{\prime}}$ for the quasivertex $\hat{V}$;

(ii) (if one removes $e$ to form a quasivertex $\hat{V}$ of $\delta \rightarrow \delta^{\prime}$ type) $\beta_{V}=$ $\alpha_{V}, \tilde{\beta_{V}}=\tilde{\alpha_{V}}$ for any vertex $V$ of $\hat{\Gamma}_{\delta}^{(e)}$ and $\beta_{\hat{V}}=\alpha_{V^{\prime}}+\mu^{-2} \alpha_{V}, \widetilde{\beta_{\hat{V}}}=$ $\tilde{\alpha}_{V^{\prime}}+\mu^{-2} \tilde{\alpha}_{V}$ for the quasivertex $\hat{V}$.

The proof of this Lemma is nothing but a slight modification of the proof of Theorem 4.1. We therefore take the liberty of omitting it.

Noting that under the additional assumption of either $\alpha_{V^{\prime}}=\tilde{\alpha}_{V^{\prime}}=0$ or $\alpha_{V}=\tilde{\alpha}_{V}=0$ either the matrix $\hat{B}_{e}$ or the matrix $\hat{\tilde{B}}_{e}$ does not depend on $\mu^{2}$, one arrives at

Theorem 4.6. Assume that $\Gamma_{\delta}$ is a graph of more than 2 vertices with all edges (except loops) of mixed type. Assume that $A_{\vec{\alpha}}$ and $A_{\overrightarrow{\tilde{\alpha}}}$ are two isospectral graph Laplacians on $\Gamma_{\delta}$. Let $V$ and $V^{\prime}$ be $\delta$ and $\delta^{\prime}$ endpoints, respectively, of an edge e. Then the graph Laplacians $A_{\overrightarrow{\alpha_{e}}}$ and $\overrightarrow{A_{\overrightarrow{\alpha_{e}}}}$ on the graph $\Gamma_{\delta}^{(e)}$ are isospectral for $\vec{\alpha}_{e}$ and $\overrightarrow{\tilde{\alpha}}_{e}$. Here

(i) if $\alpha_{V^{\prime}}=\tilde{\alpha}_{V^{\prime}}=0$, the graph $\Gamma_{\delta}^{(e)}$ is nothing but $\hat{\Gamma}_{\delta}^{(e)}$ of Definition 4. part (i), where the quasivertex $\hat{V}$ of $\delta^{\prime} \rightarrow \delta$ type is converted to a regular one by assigning the type $\delta$ to it with the coupling constant taken from the vertex $V . \vec{\alpha}_{e}$ and $\overrightarrow{\alpha_{e}}$ are constructed by dropping $\alpha_{V^{\prime}}$ and $\tilde{\alpha}_{V^{\prime}}$ from $\vec{\alpha}$ and $\overrightarrow{\tilde{\alpha}}$, respectively;

(ii) if $\alpha_{V}=\tilde{\alpha}_{V}=0$, the graph $\Gamma_{\delta}^{(e)}$ is nothing but $\hat{\Gamma}_{\delta}^{(e)}$ of Definition 4. part (ii), where the quasivertex $\hat{V}$ of $\delta \rightarrow \delta^{\prime}$ type is converted to a regular one by assigning the type $\delta^{\prime}$ to it with the coupling constant taken from the vertex $V^{\prime} . \vec{\alpha}_{e}$ and $\overrightarrow{\tilde{\alpha}}_{e}$ are constructed by dropping $\alpha_{V}$ and $\tilde{\alpha_{V}}$ from $\vec{\alpha}$ and $\overrightarrow{\tilde{\alpha}}$, respectively.

A sketch of the proof. It suffices to check the conditions of "if" implication of Theorem 3.5, i.e., to ascertain (8).

On the other hand, Lemma 4.5 yields (20), and moreover within our assumptions one clearly has $\Phi(\vec{\alpha})=\Phi_{e}\left(\overrightarrow{\alpha_{e}}\right), \Phi(\overrightarrow{\tilde{\alpha}})=\Phi_{e}\left(\overrightarrow{\tilde{\alpha}_{e}}\right)$.

Repeating the argument leading to Theorem 3.4 using Definition 5 , one by the linear independence argument, exactly as in the proof of Theorem 3.5 (in the process one surely has to redefine the function 
$\Pi(\mu)$ appropriately, see Lemma 3.1 for details), immediately obtains (8). The only part of this argument that deserves a comment is the fact that the cancellation procedure described in Definition 2 is exactly the same for $\hat{\Gamma}_{\delta}^{(e)}$ as it would be for $\Gamma_{\delta}^{(e)}$. This follows immediately from Proposition 2.1 and Definition 5.

Unfortunately, a straightforward application of this Theorem is not enough to complete the job due to the possible presence of $0 \overline{0}$ and $\overline{0} 0$ class vertices. This becomes apparent if one considers a star-graph of exactly 3 rays, one of the boundary vertices being of 00 class. The argument presented then reduces the problem to $A_{3}$ which leaves the question of isospectrality open for the named star graph. In fact, a direct application of Theorem 3.5 yields uniqueness in this case, thus necessitating further in-depth analysis of the general situation.

First we employ the results of [12] which together with Theorem 4.6 and Theorem 3.8 allow to give a complete solution of the problem of isospectrality provided that there are no vertices of $0 \overline{0}$ and $\overline{0} 0$ classes.

Theorem 4.7. Assume that $\Gamma_{\delta}$ is an arbitrary marked graph of more than two vertices. Assume that $A_{\vec{\alpha}}$ and $A_{\vec{\alpha}}$ are two isospectral graph Laplacians on $\Gamma_{\delta}$. Moreover, assume that there are no vertices $V$ of valence 2 satisfying the condition $\alpha_{V}=\tilde{\alpha}_{V}=0$. Then the following implication holds: if at a vertex $V$ one has $\alpha_{V}=\tilde{\alpha}_{V}=0$, then for every graph vertex $W$ adjacent to $V$ the identity $\alpha_{W}=\tilde{\alpha}_{W}$ holds.

Proof. Let $V$ be a vertex of 00 class. Theorem 4.1 and Theorem 4.6 justify the procedure of removing any edge $e$ incident to this vertex, be it of $\delta-\delta, \delta^{\prime}-\delta^{\prime}$ or $\delta-\delta^{\prime}$ type, with absolutely the same implications on the graph itself: the vertex $V$ joins $W$, the other endpoint of $e$, whereas the type of $W$ and the coupling constants $\alpha_{W}$ and $\tilde{\alpha}_{W}$ remain unchanged. The corresponding graph Laplacians $A_{\overrightarrow{\alpha_{e}}}$ and $A_{\overrightarrow{\alpha_{e}}}$ are isospectral.

On the other hand, the results of 12 tell us that under the condition of isospectrality vectors $\vec{\alpha}$ and $\overrightarrow{\tilde{\alpha}}$ have the same number of zero components; what's more, equal numbers of zero coupling constants pertaining to vertices of $\delta^{\prime}$ type (and hence of $\delta$ type) are also guaranteed. Moreover, as in the proof of Theorem 3.8 , one has the set equality $S(\vec{\alpha})=S(\overrightarrow{\tilde{\alpha}})$ (see (11) for the definition of the set $S$ ). In our setting, the same number of sigmas, say, $k$ is equal to zero in both $S$-sets. Let $\hat{S}$ be the set $S$, where all zero elements have been removed. Then under the condition of isospectrality $\hat{S}(\vec{\alpha})$ is nothing but a transposition of the set $\hat{S}(\overrightarrow{\tilde{\alpha}})$. 
It remains to be seen that $\sigma_{W}=\tilde{\sigma}_{W}$. Assume the opposite. Note that the procedure of removing the edge $e$ changes the valence of $W$, since $V$ is by assumption not of valence 2 . It turns out that $\hat{S}\left(\vec{\alpha}_{e}\right)$ and $\hat{S}(\overrightarrow{\tilde{\alpha}})$ are the same sets as $\hat{S}(\vec{\alpha})$ and $\hat{S}(\overrightarrow{\tilde{\alpha}})$, respectively, with the only exception: the elements $\sigma_{W}$ and $\tilde{\sigma}_{W}$ are replaced by $\kappa \sigma_{W}$ and $\kappa \tilde{\sigma}_{W}$ with $\kappa \neq 1$, respectively. The constant $\kappa$ here depends on the valences of $V$ and $W$ only.

On the other hand, $\hat{S}\left(\vec{\alpha}_{e}\right)$ and $\hat{S}\left(\overrightarrow{\alpha_{e}}\right)$ have to be transpositions of each other by isospectrality of $A_{\overrightarrow{\alpha_{e}}}$ and $A_{\overrightarrow{\alpha_{e}}}$. One easily convinces oneself that this immediately leads to a contradiction, unless $\sigma_{W}=\tilde{\sigma}_{W}$.

Remark 5. The condition of the latter Theorem that there are no 00 class vertices of valence 2 in $\Gamma_{\delta}$ can be assumed w.l.o.g. Indeed, if one has a 00 vertex in $\Gamma_{\delta}$, either of $\delta$ or $\delta^{\prime}$ type, it follows that a function in the domain of both $A_{\vec{\alpha}}$ and $A_{\overrightarrow{\tilde{\alpha}}}$ is continuous together with its first derivative through the given vertex. This means that the vertex itself can be removed from the graph (cf. [26], where this procedure is called cleaning).

It is now left to complete our analysis of graphs $\Gamma_{\delta}$ with all edges of mixed type. We will consider the situation when there exist vertices of $0 \overline{0}$ and/or $\overline{0} 0$ class.

The analysis we are about to present is probably not the simplest way of obtaining the result sought, but in our view the most transparent one. It is based on Lemma 4.5. As above, we assume w.l.o.g. that $\Gamma_{\delta}$ contains no vertices of valence 2 which are of 00 class.

We start by ascertaining the following corollary of the named Lemma. Assume that a vertex $V^{\prime}$ is of $\delta^{\prime}$ type and of $0 \overline{0}$ class. Then a direct application of Lemma 4.5 yields: any other $\delta^{\prime}$ vertex $W^{\prime}$ of the graph $\Gamma_{\delta}$ which is $\delta$-adjacent to $V^{\prime}$ (i.e., is adjacent to a $\delta$ type vertex which is in turn adjacent to $V^{\prime}$ or, in other words, is located within a single $\delta$ type vertex from $V^{\prime}$ ) is of either $\overline{0} 0$ or 00 class. Indeed, let $V^{\prime}-W-W^{\prime}$ form a chain of $\delta^{\prime}-\delta-\delta^{\prime}$ vertices. Removing an edge between $V^{\prime}$ and $W$ to form a quasivertex $\hat{V}$ of $\delta^{\prime} \rightarrow \delta$ type, use the part (i) of Lemma 4.5 and compare the terms on both sides of (20) which involve the highest power of $\mu$. These terms have to be equal due to the linear independence argument. Noting that $\hat{B}_{e}$ is independent of $\mu$, whereas $\hat{\tilde{B}}_{e}$ contains the term $\tilde{\alpha}_{V^{\prime}} \mu^{2}$ in the coupling constant pertaining to $\hat{V}$, decompose the determinant on the right hand side in the following way:

$$
\operatorname{det}\left(\hat{M}^{(e)}(\mu)-\hat{\tilde{B}}_{e}\right)=\operatorname{det}\left(\hat{M}^{(e)}(\mu)-B_{0}\right)+\operatorname{det}\left(\hat{M}_{V}^{(e)}(\mu)-B_{V}\right),
$$


where the matrices $B_{0}$ and $B_{V}$ differ only in the coupling constant pertaining to the quasivertex $\hat{V}$, this element being equal to $\tilde{\alpha}_{W}$ and $\tilde{\alpha}_{V^{\prime}} \mu^{2}$, respectively (cf. Lemma 4.5), and $\hat{M}_{V}^{(e)}(\mu)$ is $\hat{M}^{(e)}(\mu)$ with both the $\hat{V}$ th row and column zeroed out.

From Theorem 3.5 one knows that there must be a vertex of $\delta^{\prime}$ type and of $\overline{0} 0$ class in $\Gamma_{\delta}$. It then becomes apparent (cf. Definition 5) that the terms with the highest power in $\mu$ come from the second term on the r.h.s. of (21) only.

On the other hand, by Theorem 3.4 (cf. the proof of Theorem 4.6), one can single out the following subgraphs $G \in \mathcal{G}$ which are sure to yield the topmost power of $\mu$ on the l.h.s. of (20): pick an alpha-loop at every $\delta^{\prime}$ type vertex with non-zero coupling constant and a regular loop at any other vertex of the graph $\Gamma_{\delta}^{(\bmod )}$ (one uses Lemma 3.7 to ensure that subgraphs selected are in fact in $\hat{\mathfrak{G}}$ ). The second term on the r.h.s. of (21) can in turn be treated as the sum of subgraph weights over subgraphs of $\mathcal{G}$ such that the alpha-loop with the weight $\tilde{\alpha}_{V^{\prime}} \mu^{2}$ is taken at the vertex $\hat{V}$. It is now obvious that if $\tilde{\alpha}_{W^{\prime}} \neq 0$, there exists no subgraph yielding the highest power of $\mu$ and containing $\tan l_{W W^{\prime}} \mu$, where $l_{W W^{\prime}}$ is the length of the edge $W W^{\prime}$, on the r.h.s. of (20), whereas this term exists on the l.h.s. due to Lemma 3.7, unless $\Gamma_{\delta}$ is $A_{3}$. In the $A_{3}$ case however Theorem 3.5 already guarantees that $W^{\prime}$ must be of $\overline{0} 0$ class. We have therefore arrived at a contradiction.

Consideration of a vertex $V$ of $\delta^{\prime}$ type and of $0 \overline{0}$ class is done in absolutely the same way, comparing the lowest possible powers of $\mu$. One then obtains that any other $\delta$ vertex $W$ of the graph $\Gamma_{\delta}$ which is $\delta^{\prime}$-adjacent to $V$ is of either $\overline{0} 0$ or 00 class.

One can in fact do even better than that. We will argue that in the setup introduced above the vertex $W^{\prime}$ cannot be of 00 class provided that $W^{\prime} \in \partial \Gamma_{\delta}$. Indeed, having assumed the opposite, one sees by means of the same argument that the weight of any subgraph of $\mathcal{G}$ giving rise to the highest power in $\mu$ on the r.h.s. of (20) must contain $\tan l_{W W^{\prime}} \mu$, whereas there clearly exists a subgraph on the l.h.s. such that it does not. This subgraph is nothing but the 2-loop of vertices $W$ and $W^{\prime}$, built up to a spanning subgraph of $\mathcal{G}$ in a way such that its weight contains the maximal power of $\mu$ (i.e., by taking alpha-loops at all graph vertices of $\delta^{\prime}$ type with non-zero coupling constants). By linear independence argument this leads to a contradiction. Surely the same holds for a boundary vertex $W$ of $\delta$ type and of 00 class.

It is then possible, having started with a graph $\Gamma_{\delta}$ having every edge of mixed type, to proceed as follows. 
If there exists a $\delta^{\prime}$ vertex $V^{\prime}$ of $0 \overline{0}$ class, start with it. If there are no such vertices, find a $\delta$ vertex $V$ of the same class instead. If this also fails, one uses Theorem 4.7.

Starting with $V^{\prime}$, consider all $\delta$-adjacent vertices. Those of them that are internal might be of 00 class; in this case they are removed using Theorem 4.6. After this first removal, a certain number of $\delta-\delta$ edges appears in the graph, which we remove using Theorem 4.1. The procedure eventually leads to a graph $\Gamma_{\delta}^{(0)}$ which has all $\delta^{\prime}$ vertices of $0 \overline{0}$ or $\overline{0} 0$ classes and by construction has all its edges of mixed type.

Now consider every vertex $V$ of $\delta$ type to be found in the graph $\Gamma_{\delta}^{(0)}$. Our first claim is that in order to avoid a contradiction, it might be connected to at most 2 different vertices (of $\delta^{\prime}$ type). This follows from the fact obtained above that $\delta$-adjacent $\delta^{\prime}$ type vertices have to be of different classes, i.e., if one of them is $0 \overline{0}$, the other one must be $\overline{0} 0$, in conjunction with the absence of 00 class vertices of $\delta^{\prime}$ type. Moreover, a simple argument based again on Lemma 4.5 shows, that the valence of $V$ must be equal to 2 (thus, multiple edges incident to it are disallowed).

Therefore, unless we have already arrived at a contradiction, we have a graph $\Gamma_{\delta}^{(0)}$ with all $\delta^{\prime}$ vertices of either $0 \overline{0}$ or $\overline{0} 0$ class, "connected" by $\delta$ vertices of valence 2 .

This graph must contain at least a single internal $\delta^{\prime}$ vertex (otherwise the graph $\Gamma_{\delta}^{(0)}$ reduces to the chain $A_{3}$, but then clearly $\Gamma_{\delta}$ was a tree; then if $\Gamma_{\delta} \neq A_{3}$, at the penultimate step of reduction one can apply Theorem 4.6 to show that $\alpha_{V}=\tilde{\alpha}_{V}$ at the only internal vertex of $\delta$ type, which prevents isospectrality by Example 1). There are two distinct possibilities: one either has no $\delta$ type vertices of $0 \overline{0}$ class or there exists at least one.

In the former case, one can remove any of 00 classed $\delta$ vertices. We note that these vertices cannot be located on the graph boundary since otherwise Theorem 4.7 is applicable, leading to a contradiction. Removing an internal 00 vertex, one gets at least one edge $e$ connecting two vertices of $\delta^{\prime}$ type and of $0 \overline{0}$ and $\overline{0} 0$ classes, respectively. Theorem 4.1 yields that removing this edge one gets a $\overline{0} \overline{0}$ classed $\delta^{\prime}$ vertex which leads to a contradiction by the argument above, provided that $\Gamma_{\delta}^{(0)} \mathrm{had}$ more than 2 vertices of $\delta^{\prime}$ type. If on the other hand there were just 2 in $\Gamma_{\delta}^{(0)}$, one has to resort to Lemma 4.5. A straightforward but rather lengthy argument then leads to a contradiction again.

Thus, one may assume that the graph $\Gamma_{\delta}^{(0)}$ contains no 00 classed vertices of $\delta$ type. One then applies the analysis developed in the 
proof of Theorem 3.8 verbatim for an internal vertex of $\delta^{\prime}$ type. The corresponding balancing equation immediately yields a contradiction.

If on the other hand the graph does contain a $\delta$ type vertex of $0 \overline{0}$ class (and then another one of $\overline{0} 0$ class), this again yields that any $\delta^{\prime}$ adjacent $\delta$ type vertex is of either $\overline{0} 0$ or 00 class (the latter possibility being disallowed for boundary vertices). The possibility of internal 00 class $\delta$ type vertices also leads to a contradiction. Assume the opposite: let $V$ be a $\delta$ type vertex of valence 2 and of 00 class. One first removes it by Remark 5 and then uses Theorem 4.1 to get rid of the resulting $\delta^{\prime}-\delta^{\prime}$ edge, giving rise to a $\delta^{\prime}$ type vertex of $\overline{0} \overline{0}$ class. If one has at least one more $\delta$-adjacent $\delta^{\prime}$ vertex of $\overline{0} 0$ or $0 \overline{0}$ class, this turns out to be impossible. In the remaining case of just two $\delta^{\prime}$ vertices in $\Gamma_{\delta}^{(0)}$ linked together by a 00 class $\delta$ vertex, one gets to a contradiction by applying Lemma 4.5.

The graph $\Gamma_{\delta}^{(00)}$ thus obtained is clearly either a chain or a simple cycle. In both cases, it has (i) all edges of mixed type, and (ii) all vertices of either $0 \overline{0}$ or $\overline{0} 0$ class.

In the case when $\Gamma_{\delta}^{(00)}$ is a chain, a straightforward check based on Lemma 4.5 again ensures a contradiction.

In the case of a simple cycle (surely, conditions (i) and (ii) tell us that this has to be a cycle of $4 k$ vertices for natural $k$ ) one has to use Theorem 3.5. Since this graph is not a tree, Corollary 3.6 is applicable. A rather straightforward analysis now shows, that in this case nontrivial isospectral configurations are disallowed.

Ultimately, we sum up results obtained above in the form of the following

Theorem 4.8. Assume that $\Gamma_{\delta}$ is a graph which is not a chain $A_{2}$ of exactly 2 vertices with each edge (except loops) of mixed type. Assume that $A_{\vec{\alpha}}$ and $A_{\vec{\alpha}}$ are two isospectral graph Laplacians on $\Gamma_{\delta}$. If the graph contains no vertices $V$ of valence 2 such that $\alpha_{V}=\tilde{\alpha}_{V}=0$, then either $\Gamma_{\delta}=A_{3}$ of Example 1 or $\vec{\alpha}=\overrightarrow{\tilde{\alpha}}$.

Acknowledgements. The authors express deep gratitude to Prof. Sergey Naboko for constant attention to their work. We would also like to cordially thank our referees for making some very helpful comments.

\section{REFERENCES}

[1] Ashurova, E.N.; Kandagura, A.N. and Karpenko, I.I. The criterion of simplicity for symmetric operator on a graph, Methods Func. Anal. Topology, 20 (2014), no. 1, to appear.

\footnotetext{
${ }^{3}$ This assumption is w.l.o.g. by Remark 5 .
} 
[2] Avdonin S. and Kurasov P., Inverse problems for quantum trees, Inverse Problems and Imaging, 2 (2008), 1-21.

[3] Avdonin S., Kurasov P. and Nowaczyk M., Inverse Problems for Quantum Trees II. Recovering Matching Conditions for Star Graphs, Inverse Problems and Imaging, 4/4 (2010), 579-598.

[4] Belishev, M. I.; Vakulenko, A. F. Inverse problems on graphs: recovering the tree of strings by the BC-method. J. Inverse Ill-Posed Probl. 14 (2006), no. 1, $29-46$.

[5] Belishev, M. I.; Wada, N. On revealing graph cycles via boundary measurements. Inverse Problems 25 (2009), no. 10, 105011, 21 pp.

[6] Berkolaiko, G. and Kuchment, P. Introduction to Quantum Graphs, Mathematical Surveys and Monographs, vol. 186, AMS, 2012.

[7] Cassels, J. W. S. An introduction to Diophantine approximation. Cambridge Tracts in Mathematics and Mathematical Physics, No. 45. Cambridge University Press, New York, 1957. $\mathrm{x}+166 \mathrm{pp}$.

[8] Carlson, Robert. Inverse eigenvalue problems on directed graphs. Trans. Amer. Math. Soc. 351 (1999), no. 10, 4069-4088.

[9] Derkach V. A. and Malamud M. M., Generalized resolvents and the boundary value problems for Hermitian operators with gaps, J. Funct. Anal. 95 (1991), $1-95$.

[10] Ershova Yu. and Kiselev A.V., Trace formulae for graph Laplacians with applications to recovering matching conditions, Methods of Funct. Anal. Topol. 18, no. 4 (2012), 343-359.

[11] Ershova Yu. and Kiselev A.V., Trace formulae for Schrodinger operators on metric graphs with applications to recovering matching conditions, Methods of Funct. Anal. Topol. 20, no. 2 (2014), 134-148.

[12] Ershova Yu., Karpenko I.I. and Kiselev A.V., Isospectrality for graph Laplacians under the change of coupling at graph vertices, arXiv: 1405.2997 [math.sp].

[13] Exner, P., A duality between Schrödinger operators on graphs and certain Jacobi matrices, Ann. Inst. H. Poincare 66 (1997), 359-371.

[14] Exner, P., Lattice Kronig-Penney models, Phys. Rev. Lett. 74 (1995), 35033506.

[15] Gorbachuk V. I. and Gorbachuk M. L., Boundary value problems for operator differential equations. Translated and revised from the 1984 Russian original. Mathematics and its Applications (Soviet Series), 48. Kluwer Academic Publishers Group, Dordrecht, 1991.

[16] Gutkin, B. and Smilansky, U., Can one hear the shape of a graph?, J. Phys. A. 34 (2001), 6061-6068.

[17] Kostrykin V., Potthoff J. and Schrader R., Heat kernels on metric graphs and a trace formula, in "Adventures in Mathematical Physics", Contemporary Mathematics 447, Amer. Math. Soc., 2007, 175-198.

[18] Kostrykin V. and Schrader R., Kirchhoff's rule for quantum wires, J. Phys. A. 32 (1999), 595-630.

[19] Kottos, T. and Smilansky, U., Periodic orbit theory and spectral statistics for quantum graphs, Ann. Physics, 274 (1999), 76-124.

[20] Kočubeř A. N., On extension of symmetric operators and symmetric binary relations Math. Notes 17 (1975), 41-48. 
[21] Kočuber A. N., Characteristic functions of symmetric operators and their extensions (in Russian), Izv. Akad. Nauk Arm. SSR Ser. Mat. 15, 3 (1980), 219-232.

[22] Kuchment, P., Quantum graphs: an introduction and a brief survey, in "Analysis on Graphs and its Applications", Proc. Symp. Pure. Math., AMS 2008, 291-314.

[23] Kurasov P. and Nowaczyk M., Inverse spectral problem for quantum graphs, J. Phys. A: Mathematical and General, 38 (2005), 4901-4915; correction: J. Phys. A: Mathematical and General, 39 (2006), 993.

[24] Kurasov, P.; Malenová, G.; Naboko, S. Spectral gap for quantum graphs and their edge connectivity. J. Phys. A 46 (2013), no. 27, 275309, 16 pp.

[25] Kurasov P., Graph Laplacians and Topology, Arkiv för Matematik 46 (2008), 95-111.

[26] Kurasov P., Inverse problem for Aharonov-Bohm rings, Math. Proc. Cambridge Philosophical Society, 148 (2010), 331-362.

[27] Levin, B. Ya., Lectures on entire functions. In collaboration with and with a preface by Yu. Lyubarskii, M. Sodin and V. Tkachenko. Translated from the Russian manuscript by Tkachenko. Translations of Mathematical Monographs, 150. American Mathematical Society, Providence, RI, 1996. xvi +248 pp.

[28] Pivovarchik, V.; Taystruk, O. On characteristic functions of operators on equilateral graphs. Methods Funct. Anal. Topology 18 (2012), no. 2, 189-197.

[29] Roth, J.P., Le spectre du Laplacien sur un graphe, in Théorie du Potentiel (Orsay, 1983), Lect. Notes in Math., 1096, 521-539.

[30] Ryzhov V., Functional model of a class of nonselfadjoint extensions of symmetric operators, Operator Theory: Adv. Appl. 174 (2007), 117-158.

[31] Shabat, B. V. Introduction to complex analysis. Part II. Functions of several variables. Translated from the third (1985) Russian edition by J. S. Joel. Translations of Mathematical Monographs, 110. American Mathematical Society, Providence, RI, 1992. $\mathrm{x}+371 \mathrm{pp}$.

[32] Tutte, W. T. Graph theory. With a foreword by C. St. J. A. Nash-Williams. Encyclopedia of Mathematics and its Applications, 21. Addison-Wesley Publishing Company, Advanced Book Program, Reading, MA, 1984. xxi+333 pp.

Institute of Mathematics, National Academy of Sciences of Ukraine. 01601 Ukraine, Kiev-4, 3, Tereschenkivska st.

E-mail address: julija.ershova@gmail.com

Department of Algebra and Functional Analysis, V.I. Vernadsky Taurida National university. 95007 Ukraine, Autonomous Republic of Crimea, Simferopol, 4 Vernadsky pr.

E-mail address: i_karpenko@ukr.net

Department of Functional Analysis, Pidstryhach Institute for ApPlied Problems of Mechanics and Mathematics, National Academy of Sciences of Ukraine, 3-B Naukova Str. 79060, L'viv, Ukraine 
Department of Higher Mathematics and Mathematical Physics, St. Petersburg State University, 1 Ulianovskaya Street, St. Petersburg, St. Peterhoff 198504 Russia

E-mail address: alexander.v.kiselev@gmail.com 